\title{
1. A noise correction of the $\gamma$-index method for Monte Carlo dose distribution comparison
}

$4^{1}$ UCLouvain, Molecular Imaging, Radiotherapy \& Oncology, Brussels, Belgium

$5^{2}$ KU Leuven, Department of Oncology, laboratory of Experimental Radiotherapy, Leuven, 6 Belgium

7 E-mail: marie.cohilis@uclouvain.be

\begin{abstract}
Purpose: Due to the increasing complexity of IMRT/IMPT treatments, quality assurance (QA) is essential to verify the quality of the dose distribution actually delivered. In this context, Monte Carlo (MC) simulations are more and more often used to verify the accuracy of the treatment planning system (TPS). The most common method of dose comparison is the $\gamma$-test, which combines dose difference and distance-to-agreement (DTA) criteria. However, this method is known to be dependent on the noise level in dose distributions. We propose here a method to correct the bias of the $\gamma$ passing rate (GPR) induced by MC noise.

Methods: The GPR amplitude was studied as a function of the MC noise level. A model of this noise effect was mathematically derived. This model was then used to predict the time consuming low-noise GPR by fitting multiple fast MC dose calculations. MC dose maps with a noise level between $2 \%$ and $20 \%$ were computed, and the GPR was predicted at a noise level of $0.3 \%$. Due to the asymmetry of the $\gamma$-test, two different cases were considered: the MC dose was first set as reference dose, then as evaluated dose in the $\gamma$-test. Our method was applied on six proton therapy plans including analytical doses from the TPS or patient-specific QA measurements.

Results: An average absolute error of $4.31 \%$ was observed on the GPR computed for MC doses with $2 \%$ statistical noise. Our method was able to improve the accuracy of the gamma passing rate by up to $13 \%$. The method was found especially efficient to correct the noise bias when the DTA criterion is low.
\end{abstract}

Conclusions: We propose a method to enhance the $\gamma$-evaluation of a treatment plan 
when there is noise in one of the compared distributions. The method allows, in a tractable time, to detect the cases for which a correction is necessary and can improve the accuracy of the resulting passing rates.

Key words: patient QA, $\gamma$-evaluation, Monte Carlo simulation.

\section{Introduction}

Radiotherapy techniques are in constant progress to improve the radiation delivery to the target, while allowing better sparing of healthy tissues. Intensity-modulated radiotherapy (IMRT) and, more recently, intensity-modulated proton therapy (IMPT) enable the delivery of very conformal dose distributions to the target.1,2 This additional flexibility also comes with an increased complexity in the delivery equipment and treatment plan optimization algorithms. Therefore, quality assurance (QA) is essential to evaluate the quality of the treatment delivered in the patient. ${ }^{3}$ In particular, patient-specific QA (PSQA) verifies that the delivered dose corresponds to the expected dose distribution computed by the treatment planning system (TPS). This procedure generally involves the comparison of the TPS dose with experimental measurements of the delivered dose $^{4,5}$ and, sometimes, with an independent dose calculation algorithm. ${ }^{6}$

In this context, dose comparison tools are needed. Several methods have been developed over time. The dose difference (DD) criterion, the most intuitive of all the methods, proposes to evaluate the difference of dose in each voxel. The distance-to-agreement (DTA) criterion returns the minimum distance between two voxels of the same dose. ${ }^{7}$ There are then methods based on both DTA and DD criteria, such as the composite analysis ${ }^{8,9}$ or the $\gamma$-test. ${ }^{10}$ Currently, the $\gamma$-test is the most popular method for dose comparison. ${ }^{11,12} \mathrm{~A}$ passing rate is generally computed as the percentage of voxels passing this test. But although the $\gamma$-index is widely used in clinical and research environments, it has several well-known drawbacks. First of all, it is not a symmetrical test. Indeed, the results depend on which one of the compared dose distributions is set as the reference and which one is set as the evaluated dose. ${ }^{13,14}$ This is due to the fact that, for each reference dose voxel, a search is made in the evaluated dose in order to find a voxel having a dose similar to the one of the reference voxel and being spatially close enough. Besides the asymmetry issue, the $\gamma$-test also has a sensitivity to the resolution of 
the compared dose maps, especially the evaluated one. ${ }^{15,16,14,17}$ Indeed, with a low resolution for the search space of the $\gamma$-test, less voxels are within the search radius imposed by the DTA criterion. An extreme example of this situation is when voxel dimension is larger than the DTA criterion: the $\gamma$-test thus becomes a simple dose difference criterion. Finally, the presence of noise in one or both dose distributions can have an impact as well on the obtained passing rate. ${ }^{13}$ Setting the noisy dose as reference leads to an underestimated passing rate. In contrast, choosing the noisy dose as the evaluated one would provide an overestimated passing rate. This effect was theoretically proved for a simplified $1 \mathrm{D}$ case by Graves et al. ${ }^{18}$

The noise dependency of the $\gamma$-test may be problematic for QA applications. Indeed, experimental measurements with ionization chambers, radiographic films, or detector arrays generally contain a small noise component. Its impact on the $\gamma$ passing rate is often limited, but present. To a greater extent, the use of Monte Carlo algorithms as secondary dose verification introduces a more important noise in the compared distribution. Monte Carlo simulations are considered to offer the most accurate dose calculation, ${ }^{19,20,21}$ but their stochastic nature inevitably leads to a statistical noise in the results. Lowering the statistical noise to an acceptable level generally requires one to simulate a huge number of particles, increasing the computation time to an impractical degree for clinical applications. Although several authors reported this $\gamma$-index issue ${ }^{14,18,17}$ or tried to tackle it ${ }^{22}$ no tractable solution was found yet.

The objective of this paper is to address this issue by analyzing the impact of noise on $\gamma$-evaluations and then proposing a method to correct the $\gamma$ passing rate obtained from noisy distributions. To do so, the $\gamma$ passing rate is calculated for several quick Monte Carlo simulations computed with various statistical noise levels. The noise-free $\gamma$ passing rate is then estimated by fitting and extrapolating the data. This allows us to avoid the computation of too costly a Monte Carlo dose while subverting the problem of its noise. The method is illustrated on several proton therapy cases (prostate, lung, brain, liver, and H\&N) for the comparison of TPS, Monte Carlo, and measured dose distributions. 


\section{Materials and methods}

\subsection{The $\gamma$-index method}

In the $\gamma$-index method, the so-called evaluated dose and reference dose are compared through the use of a criterion made of both DD and DTA. Let $D_{\mathrm{r}}$ denote the reference dose and $D_{\mathrm{e}}$ the evaluated dose. We can therefore define the $\gamma$-value at each point $\vec{r}_{\mathrm{r}}$ of the reference dose by

$$
\gamma\left(\vec{r}_{\mathrm{r}}\right)=\min \left\{\Gamma\left(\vec{r}_{\mathrm{r}}, \vec{r}_{\mathrm{e}}\right)\right\} \forall\left\{\vec{r}_{\mathrm{e}}\right\},
$$

where

$$
\Gamma\left(\vec{r}_{\mathrm{r}}, \vec{r}_{\mathrm{e}}\right)=\sqrt{\frac{\left|\vec{r}_{\mathrm{e}}-\vec{r}_{\mathrm{r}}\right|^{2}}{\Delta d^{2}}+\left|\frac{D_{e}\left(\vec{r}_{\mathrm{e}}\right)-D_{r}\left(\vec{r}_{\mathrm{r}}\right)}{D_{r}\left(\vec{r}_{\mathrm{r}}\right)}\right|^{2} \frac{100^{2}}{\Delta D^{2}}} .
$$

$D_{\mathrm{r}}\left(\vec{r}_{\mathrm{r}}\right)$ is the value of the reference dose at voxel of coordinates $\vec{r}_{\mathrm{r}}$ and $D_{\mathrm{e}}\left(\vec{r}_{\mathrm{e}}\right)$ the value of the evaluated dose at a voxel of coordinates $\vec{r}_{\mathrm{e}}$. The variables $\Delta d$ and $\Delta D$ are the DTA and DD criteria, expressed in $\mathrm{mm}$ and \% respectively. They allow the user to fix a tolerance on the search radius and the dose tolerance respectively. Taking the example of a $3 \% / 3 \mathrm{~mm}$ criterion, a necessary condition for a voxel $\vec{r}_{\mathrm{r}}$ to pass the $\gamma$-test would be to find a voxel $\vec{r}_{\mathrm{e}}$ such that $\vec{r}_{\mathrm{e}}$ is less than $3 \mathrm{~mm}$ away from $\vec{r}_{\mathrm{r}}$ and $D_{e}\left(\vec{r}_{\mathrm{e}}\right)$ has less than $3 \%$ of dose difference with $D_{r}\left(\vec{r}_{\mathrm{r}}\right)$. The value $\Gamma\left(\vec{r}_{\mathrm{r}}, \vec{r}_{\mathrm{e}}\right)$ is thus some kind of Euclidean distance between $\left(\vec{r}_{\mathrm{r}}, D_{\mathrm{r}}\left(\vec{r}_{\mathrm{r}}\right)\right)$ and $\left(\vec{r}_{\mathrm{e}}, D_{\mathrm{e}}\left(\vec{r}_{\mathrm{e}}\right)\right)$ whose terms are normalized by a user-chosen tolerance. Therefore, if the minimum value of this variable is less than or equal to 1 , the point $\vec{r}_{\mathrm{r}}$ passes the $\gamma$-test. This test can thus be seen as the search of a point in the evaluated dose map having a dose similar, to a specified extent, to the reference dose point and being in a given spatial radius around it. At the end, a $\gamma$ passing rate (GPR) is obtained by calculating the percentage of voxels in the reference dose that have passed the test. A decision can then be made based on a clinical treshold depending on the situation. Typically, a clinical threshold of about $90 \%$ success for a $3 \% / 3 \mathrm{~mm}$ criterion is required to consider two dose distributions sufficiently similar. ${ }^{23}$ Note that the $\gamma$-index, as defined here, is local. It also exists a global version of this metric, where the dose difference is normalized by a constant dose such as the maximum reference dose or the prescribed dose.

\section{$2.2 \quad$ Numerical simulations}

For the purpose of this study, several proton treatment plans were optimized using the analytical dose calculation algorithm of a commercial TPS, providing noise-free, but approximate 
dose distributions. In addition, for one of the treatment plans, PSQA measurements were acquired in solid water with a MatriXX PT detector (IBA Dosimetry).

Commissioning and validation of the dose calculation algorithm had been previously done for the used beam lines.

In order to obtain noisy dose distributions as well, we used MCsquare, ${ }^{24}$ a benchmarked and validated open-source $\mathrm{MC}$ code. ${ }^{25,21}$ This is a fast algorithm, which allowed us to calculate many dose distributions having various (and sometimes very low) levels of statistical uncertainty. These calculations were based on the CT grid, i.e. the MC doses had the same spatial resolution as CT scans, with a voxel size varying between 0.6 and $3 \mathrm{~mm}$. For the PSQA measurement case, a CT scan of the solid water material was used.

Like for the TPS, commissioning and validation of MCsquare had been previously performed for the used beam lines.

In order to quantify the noise level of MC doses, the statistical uncertainty was computed in each voxel using the batch method. ${ }^{26,27}$ It was defined voxel-wise as the standard deviation of multiple batch doses divided by the mean dose, which corresponds to the classical relative standard deviation. A general noise level was then obtained, for each plan, by averaging these uncertainties over all pixels having a dose higher than $50 \%$ of the maximal dose. The obtained value was then multiplied by 100 in order to get a percentage. In what follows, it will be referred to as $\bar{\sigma}$.

Finally, many $\gamma$-tests had to be performed. To this end we used OpenReggui, an open-source image processing platform for applications in radiotherapy. ${ }^{28}$ The $\gamma$-index method, in this software, is implemented according to the fast algorithm proposed by Chen et al. ${ }^{29}$ All $\gamma$-tests in this study were performed over the region receiving at least $10 \%$ of the maximum TPS dose.

\subsection{Practical considerations in the $\gamma$-test}

Before using $\gamma$-tests on noisy doses, some important limitating effects should be considered. 


\subsubsection{Asymmetry}

As already stated, the $\gamma$-test is an asymmetric tool, meaning that the resulting passing rate will be different depending on which dose distribution is set as the reference. However, there is no physical or mathematical reason to define one dose over another as the reference dose, which complicates the use and the interpretation of the $\gamma$-test.

When there is noise in one of the compared dose distributions, the passing rate can be further affected. Monte Carlo algorithms produce noisy results by nature, and it is well known that their statistical uncertainty is approximately proportional to the inverse square root of the simulated particles number. ${ }^{30}$

In order to analyze the combination of both effects mentioned here above, we compared for five different cases analytical doses to Monte Carlo doses with various levels of noise, using $\gamma$-tests. These clinical cases were prostate, lung, brain, liver and H\&N tumors. $\gamma$-criteria of $2 \% / 2 \mathrm{~mm}, 3 \% / 3 \mathrm{~mm}, 4 \% / 4 \mathrm{~mm}$ and $3 \% / 1 \mathrm{~mm}$ were used. The MC and TPS doses were set both as reference and then as evaluated doses. These two possible cases will be referred to as $\mathrm{MC}_{\mathrm{ref}}$ case and $\mathrm{MC}_{\text {eval }}$ case.

\subsubsection{Spatial resolution}

It is well known that spatial resolution of compared doses affects the results of a $\gamma$-test. ${ }^{17}$ In the AAPM Task Group No. 218, the authors recommend to interpolate the evaluated dose so that its resolution is no greater than $1 / 3$ the DTA criterion. ${ }^{31}$ This is not an issue in the $\mathrm{MC}_{\text {ref }}$ case. But when a noisy dose is evaluated, the interpolation just adds meaningless points to the dose map and it affects its overall noise level. On the other hand, a direct computation of $\mathrm{MC}$ doses with a resolution as high as 1/3 the DTA criterion would not always be realistic in terms of computation time. We believe that, in the scope of our method, the interpolation of MC doses should be maintained, for both practical and common practice reasons. We thus followed AAPM recommendations by resampling evaluated doses using trilinear interpolation.

To remain rigorous, however, we propose a quick analysis on the liver case by comparing GPR for the interpolated case and the MC computation at a high native spatial resolution, 
in order to evaluate the difference induced in the $\gamma$-test in presence of noise. A resolution of $[0.39,0.39,0.5] \mathrm{mm}$ and a $\gamma$-criterion of $2 \% / 2 \mathrm{~mm}$ are used. We also show the impact of the resolution on the $\gamma$-index by observing GPR curves as a funtion of MC noise for various resolutions.

\subsubsection{Normalization of the dose}

In this work, and more specifically in the proposed method, only local $\gamma$-tests are performed. However, all presented results could certainly be extrapolated to the global case. The impact of noise on global GPR should be nevertheless studied beforehand. We thus propose here a comparison of the impact of noise on GPR for local and global $\gamma$-tests. To this end, we recomputed passing rates obtained with a global $\gamma$-index for patients in which we previously observed important noise impact (see Section 2.3), i.e., lung for the $\mathrm{MC}_{\text {ref }}$ case and prostate for $\mathrm{MC}_{\text {eval }}$ case. Doses were normalized to the prescription.

\subsection{An adapted use of the $\gamma$-index method}

In order to reduce the effect of noise on the $\gamma$-test, we now propose a method enabling the prediction of the GPR for a low noise MC dose (long computation time) by only computing multiple GPR obtained with various noisy MC doses (short computation time). It consists of a curve fitting followed by an extrapolation: a function linking the GPR to the statistical uncertainty is first derived through an optimization, then this function is evaluated at a low noise level in order to correct the biased GPR.

In practice, seven points $\left(\bar{\sigma}_{i}, \operatorname{GPR}\left(\bar{\sigma}_{i}\right)\right), i=1, \ldots, 7$, are computed, where $\operatorname{GPR}\left(\bar{\sigma}_{i}\right)$ represents the passing rate obtained with a MC dose having a statistical uncertainty $\bar{\sigma}_{i}$. In clinics, MC simulations are typically performed with a statistical level of $1 \%-2 \%$. Therefore, we chose here to experiment two different cases: either the seven GPR are computed for uncertainties between $1 \%$ and $20 \%$, or between $2 \%$ and $20 \%$. We thus aim at a gain in precision and not in computation time. The smallest statistical uncertainty used (1\% or $2 \%)$ will be denoted in what follows as $\bar{\sigma}_{\text {min }}$, while the highest one $(20 \%)$ will be denoted as $\bar{\sigma}_{\max }$. The seven doses needed are extracted at various stages of a single Monte Carlo simulation and do not require additional computation time. Once the $\gamma$-tests are performed, a weighted least-squares curve fitting is performed in order to predict the passing rate at a given uncertainty. This 
uncertainty must be chosen low enough so that the corresponding GPR can be considered as a reference "denoised" GPR. The value $0.3 \%$ was chosen here in order to keep tractable MC computation times during validation. The fitting model is the same for $\mathrm{MC}_{\mathrm{ref}}$ and $\mathrm{MC}_{\text {eval }}$ cases and has seven parameters given by the vector $\boldsymbol{c}=\left[c_{1}, c_{2}, c_{3}, c_{4}, c_{5}, c_{6}, c_{7}\right]$. It is given by

$$
F(\boldsymbol{c} \mid \bar{\sigma})=c_{1}+c_{6}\left(c_{3} \operatorname{erf}\left(\frac{c_{3}}{\bar{\sigma}}\right)+\frac{\bar{\sigma}}{\sqrt{\pi}} \mathrm{e}^{-\left(\frac{c_{3}}{\bar{\sigma}}\right)^{2}}-c_{2} \operatorname{erf}\left(\frac{c_{2}}{\bar{\sigma}}\right)-\frac{\bar{\sigma}}{\sqrt{\pi}} \mathrm{e}^{-\left(\frac{c_{2}}{\bar{\sigma}}\right)^{2}}\right)
$$

$$
-c_{7}\left(c_{5} \operatorname{erf}\left(\frac{c_{5}}{\bar{\sigma}}\right)+\frac{\bar{\sigma}}{\sqrt{\pi}} \mathrm{e}^{-\left(\frac{c_{5}}{\bar{\sigma}}\right)^{2}}-c_{4} \operatorname{erf}\left(\frac{c_{4}}{\bar{\sigma}}\right)-\frac{\bar{\sigma}}{\sqrt{\pi}} \mathrm{e}^{-\left(\frac{c_{4}}{\bar{\sigma}}\right)^{2}}\right)
$$

and was found to be a (parametrized) approximate upper bound of the the theoretical GPR evolution as a function of MC noise. The choice of this model is discussed in Appendix A. To perform the fit, the Nelder-Mead direct search algorithm implemented in MATLAB is used. This method was chosen for its efficiency and its calculation speed. But due to the flexibility of our model, which has many parameters, the sensitivity to initial conditions can be quite high in the optimization process. For this reason, we added constraints and made the optimization algorithm explore several initial conditions in order to avoid local minima. The solution for which the objective function is the lowest is then automatically selected. Each optimization being very fast, the entire process takes less than 10 seconds.

As the Nelder-Mead method is an unconstrained optimization algorithm, we modified the objective function

$$
F_{\text {unconstr }}(\boldsymbol{c} \mid \bar{\sigma})=\frac{1}{7} \sum_{j=1}^{7} w_{j}\left(F\left(\boldsymbol{c} \mid \bar{\sigma}_{j}\right)-G P R\left(\bar{\sigma}_{j}\right)\right)^{2}
$$

in order to allow for $n$ constraints $g_{i}(\boldsymbol{c} \mid \bar{\sigma})<0$, using a penalty function $p(\boldsymbol{c} \mid \bar{\sigma})$. This way, the new objective function to be optimized is

$$
F_{\text {constr }}(\boldsymbol{c} \mid \bar{\sigma})=F_{\text {unconstr }}(\boldsymbol{c} \mid \bar{\sigma})+p(\boldsymbol{c} \mid \bar{\sigma})
$$

where the penalty function, weighted by factors $\alpha_{i}, i=1, \ldots, n$, is given by

$$
p(\boldsymbol{c} \mid \bar{\sigma})=\sum_{i=1}^{n} \alpha_{i} \max \left\{0, g_{i}(\boldsymbol{c} \mid \bar{\sigma})\right\}^{2} .
$$

Each of the studied cases $\mathrm{MC}_{\mathrm{ref}}$ and $\mathrm{MC}_{\text {eval }}$ lead to different constraints. The penalty function $p$ is described hereafter for both cases. 


\subsubsection{Monte Carlo dose set as reference dose}

Various constraints for the optimization could be selected. In 2013, Graves et al. ${ }^{18}$ performed an analysis of the impact of noise on the GPR. Their theoretical conclusion was that in presence of noise in the reference dose, the passing rate was always underestimated. They also numerically validated that result by drawing passing rates as a function of noise for two different patients. Relying on their results, but also on ours (Section 3.1), we chose here to add three different penalties to our objective function, corresponding to three different constraints:

- The maximum predicted GPR should be $100 \%$.

- The derivatives should be negative over the extrapolated part of the curve.

This constraint arises from the fact that the GPR decreases when noise increases.

- The second derivatives should be negative or close to zero over the extrapolated part of the curve.

This constraint is derived from our results and observations of Graves et al.'s results.

Taking these constraints into account, the penalty term is finally chosen as

$$
\begin{aligned}
p(\boldsymbol{c} \mid \bar{\sigma})= & \frac{1}{l}\left(100 \max \left\{0, F\left(\boldsymbol{c} \mid 0.05 \leq \bar{\sigma} \leq \bar{\sigma}_{\min }+1\right)-100\right\}^{2}\right. \\
& +40 \max \left\{0, \frac{\partial F}{\partial \bar{\sigma}}\left(\boldsymbol{c} \mid 0.05 \leq \bar{\sigma} \leq \bar{\sigma}_{\min }+1\right)\right\}^{2} \\
& +20 \max \left\{0, \frac{\partial^{2} F}{\partial \bar{\sigma}^{2}}\left(\boldsymbol{c} \mid 0.05 \leq \bar{\sigma} \leq \bar{\sigma}_{\min }+1\right)-0.5\right\}^{2} \\
& \left.+20 \max \left\{0,-\frac{\partial^{2} F}{\partial \bar{\sigma}^{2}}\left(\boldsymbol{c} \mid 0.05 \leq \bar{\sigma} \leq \bar{\sigma}_{\min }+1\right)-4\right\}^{2}\right)
\end{aligned}
$$

where $l$ is the number of points in which constraints are evaluated and the $\alpha_{i}$ in Eq. (6) are chosen to emphasize the most important penalties.

\subsubsection{Monte Carlo dose set as evaluated dose}

With similar reasoning as for the $\mathrm{MC}_{\text {ref }}$ case, the following constraints are applied during the optimization process of the $\mathrm{MC}_{\text {eval }}$ case:

- The maximum predicted GPR should be $100 \%$.

- The derivatives should be positive over the extrapolated part of the curve. 
- The second derivatives should be negative or close to zero over the extrapolated part of the curve.

These constraints lead us to a final penalty term equal to the one given in Eq. (7), except for the sign of the first derivatives that need to be positive and not negative, resulting in a change of sign in the second constraint.

\subsection{Application and evaluation of the method}

Our method is tested on 9 proton therapy plans. The five first ones are the ones already mentioned in Section 2.3. To these are added other brain, lung and H\&N cases, along with the previously mentioned PSQA case (lung). For this last plan, one $\gamma$-test between the measurement and $\mathrm{MC}$ dose was performed for each of its two beams (left posterior oblique (LPO) and posterior (POST)). As measurements only provide $2 \mathrm{D}$ information, the $\mathrm{MC}_{\text {eval }}$ case leads to 2D/3D $\gamma$-tests since the search can be done in 3D around the slice corresponding to the measurement. In contrast, for the $\mathrm{MC}_{\mathrm{ref}}$ case, $\gamma$-tests are $2 \mathrm{D} / 2 \mathrm{D}$ since it would make no sense to use a whole 3D MC volume as a reference when all we have is a $2 \mathrm{D}$ evaluated dose, corresponding to a single slice of the reference dose. In the latter case, the slice of the MC dose is selected so that it corresponds to the theoretical measurement depth. For the other plans, 3D TPS and MC doses are compared. $\gamma$-criteria of $2 \% / 2 \mathrm{~mm}, 3 \% / 3 \mathrm{~mm}$, $4 \% / 4 \mathrm{~mm}$ and $3 \% / 1 \mathrm{~mm}$ are used.

Having at our disposal GPR corresponding to MC statistical noises of $1 \%, 2 \%, 4 \%, 7 \%$, $8 \%, 13 \%$ and $20 \%\left(\bar{\sigma}_{\min }=1 \%\right)$ or $2 \%, 3 \%, 4 \%, 7 \%, 8 \%, 13 \%$ and $20 \%\left(\bar{\sigma}_{\min }=2 \%\right)$, the curve fittings were done by giving more weight to low noise passing rates $(w=[40,30,20,10,5,1,1])$.

As explained in Section 2.4, to avoid local minima, the optimization algorithm explores multiple solutions by randomly sampling initial conditions several times. Due to this random sampling, the optimization results may vary from one run to another. In order to evaluate this statistical deviation, we applied our method 60 times for each case and report here the average predicted passing rates. Along with their standard deviation, the GPR mean absolute error with respect to the $0.3 \%$ uncertainty dose is also given, as well as the gain obtained with 
our method which was defined as

$$
\text { Gain }=\left|\mathrm{GPR}_{\text {true }, 0.3 \%}-\mathrm{GPR}_{\text {true }, \bar{\sigma}_{\text {min }}}\right|-\left|\mathrm{GPR}_{\text {true }, 0.3 \%}-\mathrm{GPR}_{\text {predicted }, 0.3 \%}\right|
$$

where GPR $\mathrm{true}, 0.3 \%_{\text {is }}$ the calculated GPR for $0.3 \%$ of statistical uncertainty, GPR ${\text { true }, \bar{\sigma}_{\text {min }}}_{\text {is the }}$ calculated passing rate obtained for the fitting point having the smallest uncertainty among all,

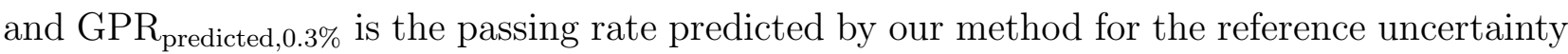
$0.3 \%$. This gain therefore represents the difference between the errors on the passing rate obtained with the classical $\gamma$-index (with a MC uncertainty $\bar{\sigma}_{\min }$ ) and with our method, if we consider the GPR for a noise level of $0.3 \%$ as being the true GPR. A positive gain therefore corresponds to an improvement by our method compared to the classical $\gamma$-index where the MC dose is calculated for a noise level $\bar{\sigma}_{\text {min }}$, while a negative gain would indicate a worsened situation.

\section{Results}

\subsection{Practical considerations in the $\gamma$-test}

\subsubsection{Asymmetry and noise impact}

The asymmetry issue of the $\gamma$-test is illustrated on Figure 1 for all five patients, with a $\gamma$-criterion of $2 \% / 2 \mathrm{~mm}$. The figure shows the passing rate curves obtained as a function of the noise level in the MC dose for both possible cases: MC dose defined as reference or as evaluated dose. Brain and $\mathrm{H} \& \mathrm{~N}$ cases, particularly, show that $\mathrm{MC}_{\text {eval }}$ and $\mathrm{MC}_{\text {ref }}$ curves do not tend to a same GPR value as the uncertainty tends to zero.

Figure 2 summarizes the error made on the GPR for all $\gamma$-criteria and five patients, according to the status of the MC dose and its level of noise. This error is defined as the difference between the GPR for a given $\bar{\sigma}\left(\mathrm{GPR}_{\bar{\sigma}}\right)$ and the GPR for the lowest available uncertainty $\left(\mathrm{GPR}_{\bar{\sigma}_{0}}\right)$. A more detailed table with all results is also shown in Appendix B (Table 1).

For the case where the Monte Carlo dose was defined as the reference dose in the $\gamma$-test, the effect of GPR underestimation due to noise presence is clearly observed; the GPR curves in Figure 1 are increasing when the statistical uncertainty decreases and are very similar, irrespective of the patient and the criterion. However, the magnitude of the underestimation varies a lot. For a noise level of 1\%, GPR underestimation can be negligible (below 1\%) or very important (going up to $8 \%$ ), depending on the patient and the criterion. For a noise 

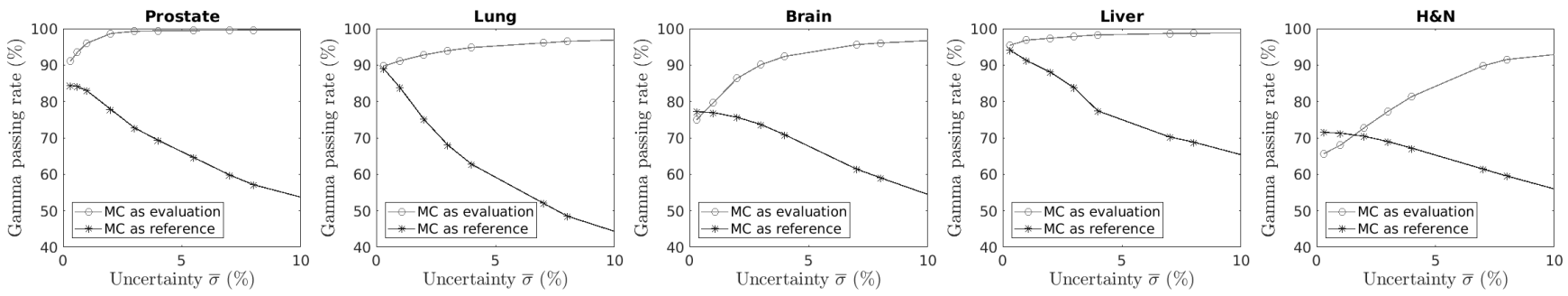

Figure 1: Gamma passing rate for $2 \% / 2 \mathrm{~mm}$ as a function of mean statistical uncertainty, for prostate, lung, brain, liver and $\mathrm{H} \& \mathrm{~N}$ cases (from left to right). The darker curve shows the case where MC dose is set as reference while the lighter one shows the case where TPS dose is set as reference.
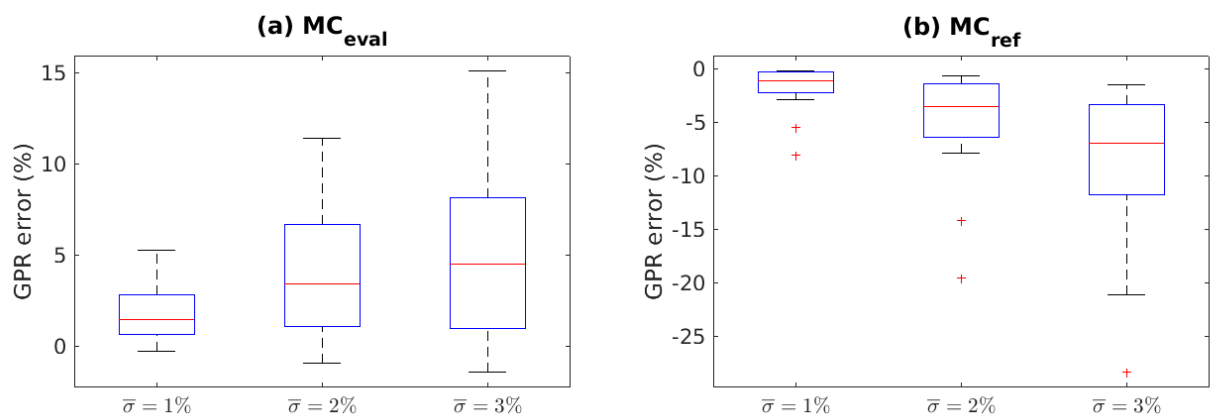

Figure 2: GPR errors summarized for five patients (prostate, lung, brain, liver, $\mathrm{H} \& \mathrm{~N}$ ) and four $\gamma$-criteria $(2 \% / 2 \mathrm{~mm}, 3 \% / 3 \mathrm{~mm}, 4 \% / 4 \mathrm{~mm}, 3 \% / 1 \mathrm{~mm})$, for (a) $\mathrm{MC}_{\text {eval }}$ case and (b) $\mathrm{MC}_{\text {ref }}$ case.

level of $2 \%$, it can even go up to almost $20 \%$. Generally, the smaller the DTA criterion, the stronger the impact of noise, which makes sense since the $\gamma$-index metric is made of a DD criterion plus a DTA criterion intended to cut some slack.

For the case where the Monte Carlo distribution is the evaluated dose in the $\gamma$-test, the effect of GPR overestimation due to noise is clear as well. However, the effect is limited when compared doses are very similar, since the maximum possible GPR is $100 \%$. This is why, for some cases, the impact of noise is very small. The maximum GPR overestimation over all five patients and criteria reaches $5.27 \%$ for $\bar{\sigma}=1 \%$ and $11.38 \%$ for $\bar{\sigma}=2 \%$.

A more detailed analysis of the noise impact is given in Appendix B. We discuss there what might affect the magnitude of GPR error due to noise.

\subsubsection{Spatial resolution}

Figure 3 shows GPR curves for various resolutions of the evaluated dose, in the liver case. The DTA criterion being here $1 \mathrm{~mm}$, only the highest resolution considered $([0.29,0.29$, 
$0.33 \mathrm{~mm}$ ) fits in the criteria of AAPM TG-218. ${ }^{31}$ According to Figure 3, it seems justified to require such a high resolution, especially in presence of noise. Notice that with the lowest resolution used here, the DTA has no impact at all since it is smaller than the voxel size in each direction. If we do not consider this curve, low noise GPR are actually quite similar for all higher resolutions, i.e., the impact of resolution is still present but minimal.

Figure 4 shows GPR evolutions for the liver $\mathrm{MC}_{\text {eval }}$ case, comparing two different $\gamma$-index computations: either the evaluated (MC) dose is interpolated to a resolution of $[0.39,0.39$, $0.5] \mathrm{mm}$, or it is directly computed with this resolution. For each considered $\gamma$-criterion, we observe a similar behavior: the interpolation results in a slightly lower GPR, regardless of the noise level.

\subsubsection{Normalization of the dose}

Figure 5 shows the GPR evolution as a function of MC noise for various cases: Figure 5(a) is the $\mathrm{MC}_{\text {ref }}$ lung case, comparing global and local $\gamma$-tests; Figure 5(b) shows the same but for
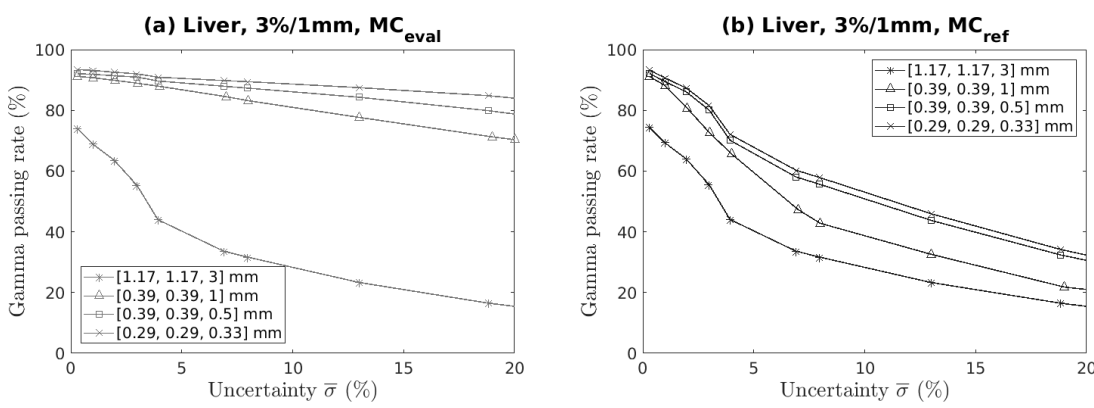

Figure 3: Comparison of several spatial resolutions of the evaluated dose for $3 \% / 1 \mathrm{~mm}$ GPR as a function of mean statistical uncertainty in the liver case, for (a) $\mathrm{MC}_{\text {eval }}$ case and (b) $\mathrm{MC}_{\text {ref }}$ case.
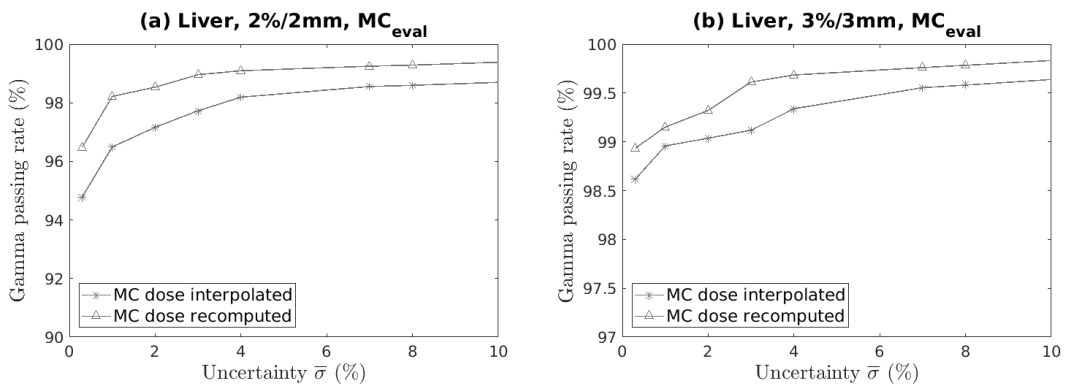

Figure 4: Comparison of MC evaluated doses interpolated and directly computed at desired resolution in the liver case, for (a) $2 \% / 2 \mathrm{~mm}$ GPR and (b) $3 \% / 3 \mathrm{~mm}$ GPR. Resolution of the MC dose is $[0.39,0.39,0.5]$. 

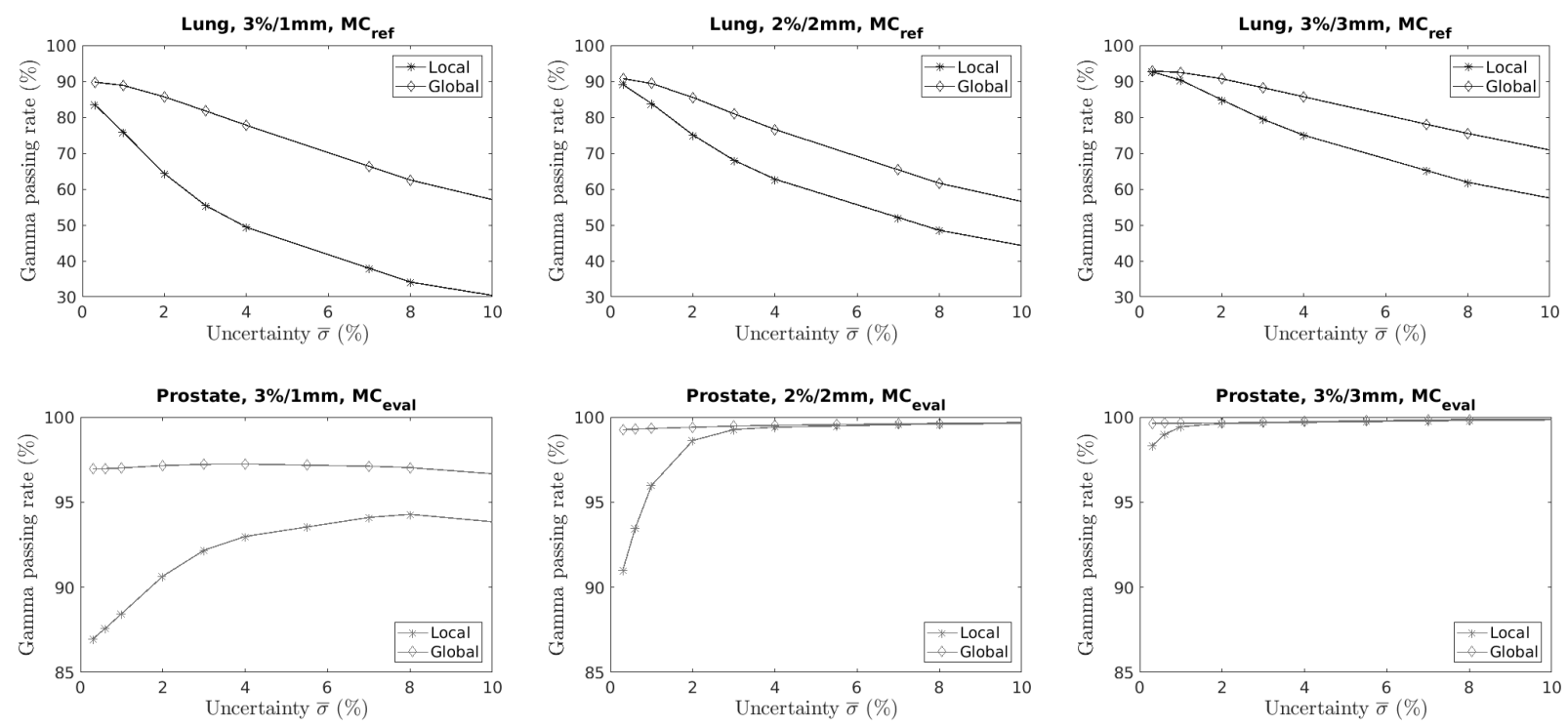

Figure 5: Comparison of global and local $\gamma$-tests: GPR as a function of mean statistical uncertainty, for lung (above) when MC dose is set as reference and prostate (below) when MC dose is evaluated $(3 \% / 1 \mathrm{~mm}$, $2 \% / 2 \mathrm{~mm}, 3 \% / 3 \mathrm{~mm}$ from left to right).

the $\mathrm{MC}_{\text {eval }}$ case with the prostate patient.

The choice of patients for this analysis was based on the importance of the GPR error observed for given noise levels. We indeed observed in Section 3.1.1 that our lung patient shows an important underestimation of the local GPR in the $\mathrm{MC}_{\text {ref }}$ case while the prostate patient leads to high GPR overestimation in the $\mathrm{MC}_{\text {eval }}$ case. We selected this way the "worst cases".

As we could expect, since the dose difference is normalized by a high constant dose in the $\gamma$-metric, we observe in Figure 5 that a global $\gamma$-index metric always leads to a better GPR. Moreover, it lessens the impact of noise. For the $\mathrm{MC}_{\text {eval }}$ case, there is almost no GPR difference between various low noise levels such as $0.3 \%, 1 \%$ or $2 \%$. For the $\mathrm{MC}_{\text {ref }}$ case, the GPR underestimation for noise levels of $1 \%$ or $2 \%$ can reach $4.1 \%$. This value remains nevertheless way below GPR errors observed for local $\gamma$-metrics.

\subsection{An adapted use of the $\gamma$-index method}

\subsubsection{Monte Carlo dose set as reference dose}

Figure 6 illustrates the method by showing a resulting fit along with the absolute value of the error in each point for the case Lung 1 and each of the four $\gamma$-criteria considered. The figure is zoomed over a range of uncertainties between $0.3 \%$ and $20 \%$, in semi-logarithmic scale. 

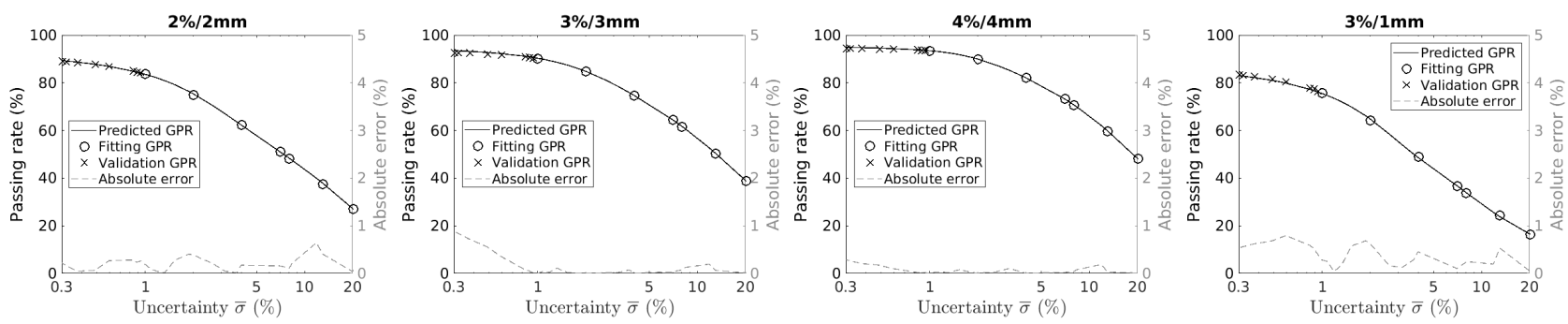

Figure 6: $\mathrm{MC}_{\text {ref }}$ case : resulting fitting and corresponding absolute error for the case Lung 1 with $\gamma$-criterion $2 \% / 2 \mathrm{~mm}, 3 \% / 3 \mathrm{~mm}, 4 \% / 4 \mathrm{~mm}$ and $3 \% / 1 \mathrm{~mm}$, when $\bar{\sigma}_{\min }=1 \%$. The circles show the points used for fitting, the crosses show the true passing rates used to validate the prediction, the plain curve shows the predicted passing rate and the dashed line the absolute error.

Figure 7 summarizes the results obtained over all nine patients (10 $\gamma$-tests with the PSQA measurements case) and $\gamma$-criteria when $\bar{\sigma}_{\min }=1 \%$. Figure $7(\mathrm{a})$ compares the GPR mean errors before and after applying the method while Figure $7(\mathrm{~b})$ shows the resulting mean gains. We see that our method allowed us to strongly reduce GPR errors in such a way that most of them are below 1\%. Moreover, the maximum error goes down from $8.03 \%$ to $1.96 \%$. Gains are mostly between $0 \%$ and $2 \%$. However, we understand from the few high gains that the method successfully corrected the highest GPR errors. Ssome negative gains are also observed, going down to $-0.87 \%$.

Similarly to Figure 7, Figure 8 summarizes the results obtained over all patients and $\gamma$-criteria when $\bar{\sigma}_{\min }=2 \%$. We see this time that new GPR errors are mostly between $0 \%$ and $3 \%$. The maximum error is reduced from $19.53 \%$ to $6.37 \%$. It remains an important error, however it corresponds to a gain of $12.96 \%$. Other gains are mostly between $0 \%$ and $7 \%$.
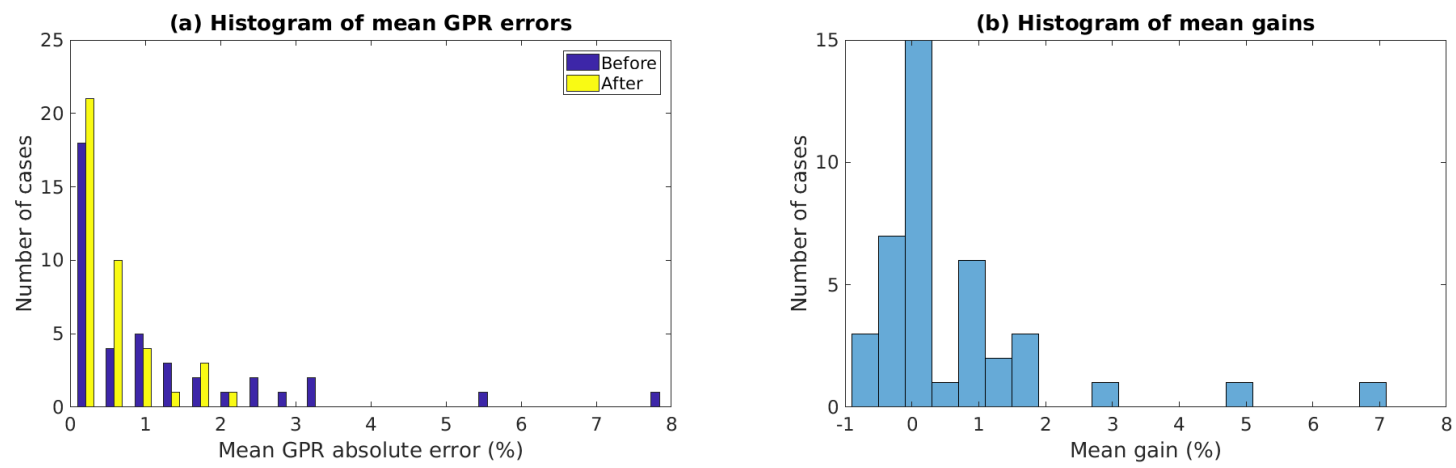

Figure 7: Summary of the results using the adapted $\gamma$-index in the $\mathrm{MC}_{\text {ref }}$ case with $\overline{\boldsymbol{\sigma}}_{\text {min }}=\mathbf{1} \%$, for all 9 patients and $\gamma$-criteria: (a) repartition of mean absolute errors, computed before and after applying the method and (b) repartition of mean gains. 

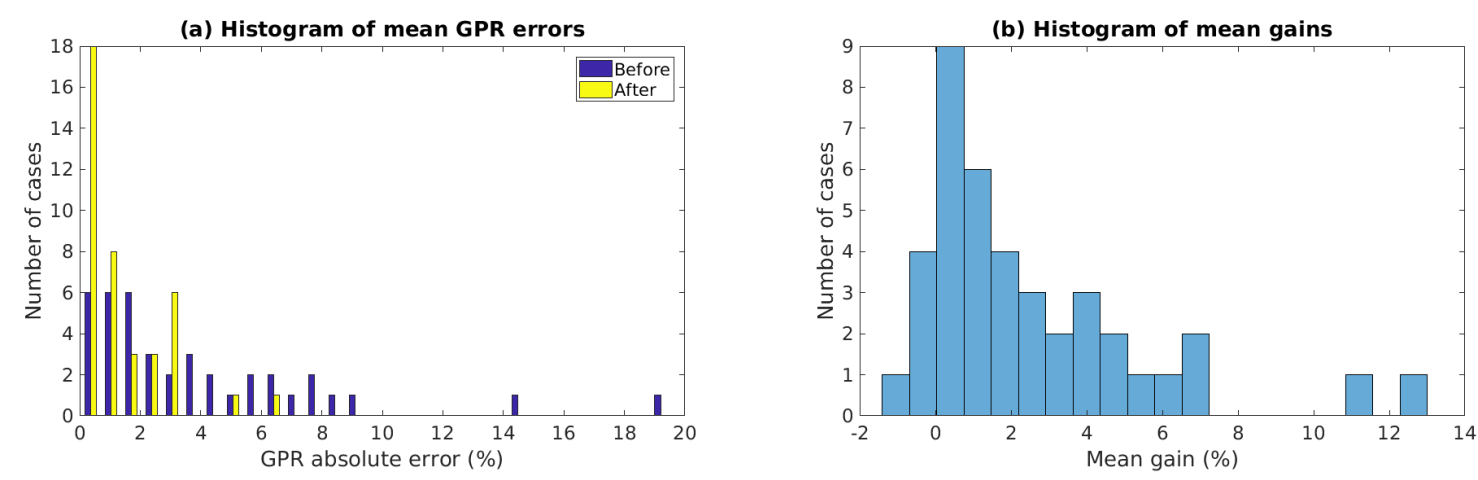

Figure 8: Summary of the results using the adapted $\gamma$-index in the $\mathrm{MC}_{\text {ref }}$ case with $\overline{\boldsymbol{\sigma}}_{\text {min }}=\mathbf{2} \%$, for all 9 patients and $\gamma$-criteria: (a) repartition of mean absolute errors, computed before and after applying the method and (b) repartition of mean gains.

More detailed results are given in Appendix C. They provide GPR for $0.3 \%$ and $\bar{\sigma}_{\text {min }}$, mean GPR obtained, mean errors, mean gains and corresponding standard deviations per patient and per $\gamma$-criterion, including for the PSQA measurement case.

\subsubsection{Monte Carlo dose set as evaluated dose}

Figure 9 shows a resulting fit along with the absolute error in each point for the case $\mathrm{H} \& \mathrm{~N} 1$ and each of the four considered $\gamma$-criteria, in semi-logarithmic scale. We can see in this case that the fitting worked quite well.

Figure 10(a) shows the GPR errors before and after applying our method, for $\bar{\sigma}_{\text {min }}=1 \%$. All nine patients and $\gamma$-criteria are included. Figure 10(b) gives the corresponding gains obtained with the proposed method. We can observe that the repartition of errors after
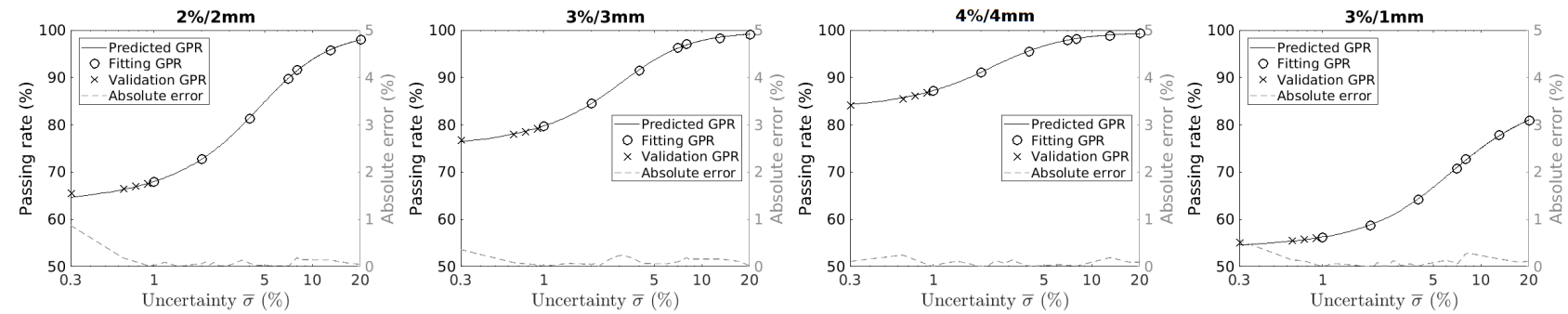

Figure 9: $\mathrm{MC}_{\text {eval }}$ case : resulting fitting and corresponding absolute error for the $\mathrm{H} \& \mathrm{~N}$ case with $\gamma$-criterion $2 \% / 2 \mathrm{~mm}, 3 \% / 3 \mathrm{~mm}, 4 \% / 4 \mathrm{~mm}$ and $3 \% / 1 \mathrm{~mm}$, when $\bar{\sigma}_{\min }=1 \%$. The circles show the points used for fitting, the crosses show the true passing rates used to validate the prediction, the plain curve shows the predicted passing rate and the dashed line the absolute error. 
applying our method is concentrated around zero. Most of them are below $1 \%$ and the maximum one is $1.89 \%$. However, the initial impact of noise was less important than in the $\mathrm{MC}_{\text {ref }}$ case, which leads to gains no higher than $3 \%$.

Similarly to Figure 10, Figure 11 summarizes the results obtained over all nine patients and $\gamma$-criteria when $\bar{\sigma}_{\min }=2 \%$. We can see here again that our method allows us to reduce GPR errors down to $2 \%$ in most cases, with a maximum error of $6.24 \%$ instead of $11.38 \%$. Most gain are between $0 \%$ and $3 \%$ but can go up to almost $10 \%$.

Similarly to the $\mathrm{MC}_{\text {ref }}$ case, more detailed results are given in Appendix $\mathrm{C}$.
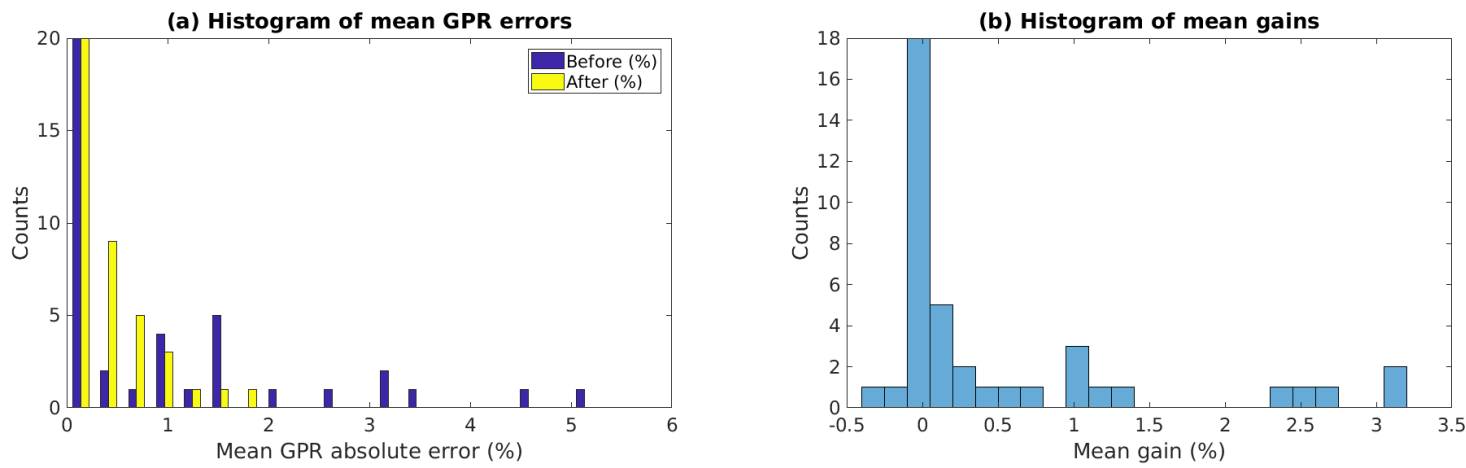

Figure 10: Summary of the results using the adapted $\gamma$-index in the $\mathrm{MC}_{\text {eval }}$ case with $\overline{\boldsymbol{\sigma}}_{\text {min }}=\mathbf{1} \%$, for all 9 patients and $\gamma$-criteria: (a) repartition of mean absolute errors, computed before and after applying the method and (b) repartition of mean gains.
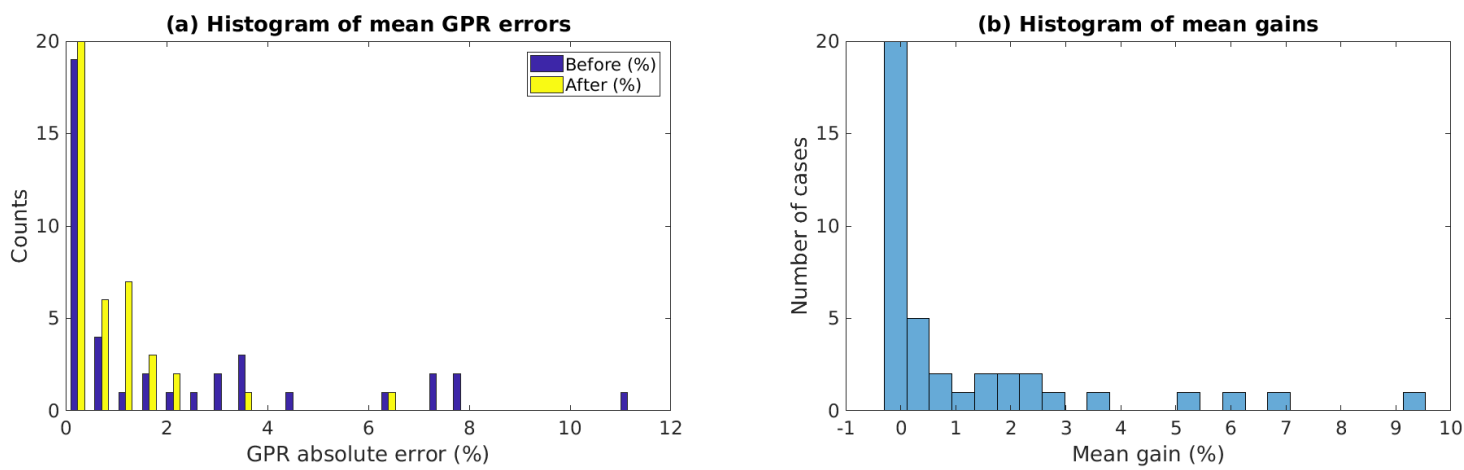

Figure 11: Summary of the results using the adapted $\gamma$-index in the $\mathrm{MC}_{\text {eval }}$ case with $\overline{\boldsymbol{\sigma}}_{\text {min }}=\mathbf{2} \%$, for all 9 patients and $\gamma$-criteria: (a) repartition of mean absolute errors, computed before and after applying the method and (b) repartition of mean gains. 


\section{Discussion}

As already mentioned in several papers, ${ }^{13,14,18,17}$ the noise in dose distributions can heavily impact the results of a dose comparison performed with the $\gamma$-index method. As shown in Section 3.1, noise in the reference dose results in an underestimated passing rate. In contrast, noise in the evaluated dose typically produces an overestimated passing rate. In our study, for a classical 3\%/3mm criterion, the GPR underestimation between $1 \%$ and the smallest available uncertainty for the $\mathrm{MC}_{\text {ref }}$ case could go up to $8.03 \%$. Moreover, a smaller DTA criterion usually led to a higher error, which makes sense since the $\gamma$-index metric is made of a DD criterion plus a relaxing DTA criterion. For the $\mathrm{MC}_{\text {eval }}$ case, the GPR error went up to $5.27 \%$.

These errors being nevertheless very patient-specific, it is difficult to evaluate the accuracy of a given $\gamma$-test. We did not find here (see Appendix B) any clear criterion to determine the severity of noise impact. Although there might be a slight dependence on the true passing rate, this value is normally not known in practice. Note that this trend was also observed by Huang et al. ${ }^{17}$ for a global $\gamma$-index. A feature not studied in this paper is the proportion of high dose gradients in the compared doses. Low and Dempsey showed in their paper of 2003 that high gradients regions in the doses led to less impact of noise on the $\gamma$-metric. ${ }^{13}$ However, this does not provide any straightforward way to quantify noise effect on the GPR. A more practical way to determine if a $\gamma$-evaluation is impacted by noise could be to perform $\gamma$-tests for two different (and close) noise levels, such as $1 \%$ and $2 \%$ or $2 \%$ and $3 \%$, to then compute the difference between obtained GPR. This could already give a good idea of the magnitude of the GPR error.

Another factor that can impact the $\gamma$ passing rate and was discussed here is the spatial resolution of the evaluated dose distribution. Indeed, we should in theory have a continuous search space when performing a $\gamma$-test. But due to the discrete nature of the dose image, this is not the case and the evaluated dose thus needs to be interpolated. The current recommendation, from AAPM TG-218, ${ }^{31}$ is to always keep its resolution below $1 / 3$ of the DTA criterion. In this paper, we compared GPR as a function of the noise level for various resolutions and observed that a coarser resolution worsened the impact of noise. The effect of interpolating a noisy (evaluated) dose was also studied. It was found to generate slightly lower GPR for $\gamma$-criteria such as $2 \% / 2 \mathrm{~mm}$ and $3 \% / 3 \mathrm{~mm}$. This can be explained by the fact 
that the interpolation process has for secondary effect to smooth the dose and therefore, the estimated noise level is too high. Therefore, the effect observed is mainly the GPR overestimation in presence of noise in the evaluated dose, although part of it might also be due to the interpolation itself, which generates artificial dose values. When decreasing the DTA criterion down to $1 \mathrm{~mm}$ (see Appendix B), however, a different effect was observed. It resulted in a decreasing GPR instead of an increasing one as when the MC dose is computed with a high native resolution. Although only one patient was considered here, we believe that resampling noisy doses should be avoided when possible.

A last feature that needs to be considered to enable a practical use of the $\gamma$-metric in presence of noise is the dose normalization, i.e., the global/local nature of the $\gamma$-test. As expected, since the dose difference is normalized by a high constant dose in the $\gamma$-metric, it was found here that noise impact is least when normalization is performed globally. For a MC dose having a noise level of $1 \%$ or $2 \%$, it becomes essentially negligible.

In order to overcome these issues of the $\gamma$-test for the comparison of MC doses and noise-free doses, we introduced a correction which allowed us to take noise in consideration. The proposed method is an extrapolation based on the computation of several $\gamma$-tests of multiple MC doses having various high levels of statistical uncertainty, the lowest being denoted as $\bar{\sigma}_{\text {min }}$. A more precise GPR can this way be obtained by extrapolation without increasing the $\mathrm{MC}$ computation time. The method was considered for both $\mathrm{MC}_{\mathrm{ref}}$ and $\mathrm{MC}_{\text {eval }}$ cases.

When we set the $\mathrm{MC}$ dose as reference in the $\gamma$-tests for a $\bar{\sigma}_{\min }$ of $1 \%$, good results are achieved by our method. Mean GPR errors, initially between $0 \%$ and $8 \%$, are reduced down to maximum $2 \%$, leading to gains up to $7 \%$. Standard deviations are very low (see Appendix C). However, the method does not systematically allow for an improvement because the GPR, at $1 \%$ of uncertainty, is already sometimes very close to the true GPR. This brings up some negative gains, which remain nevertheless of weak magnitude and correspond to small GPR errors, both initially and after application of our method.

When applying the adapted $\gamma$-index with a $\bar{\sigma}_{\text {min }}$ equal to $2 \%$, mean GPR errors go down from maximum $19 \%$ (for a regular $\gamma$-test) to maximum $6 \%$, most of them being actually between 
$0 \%$ and $3 \%$. Thus, using the method for a higher $\bar{\sigma}_{\min }$ still enables a strong reduction of GPR errors but does not guarantee such a good GPR accuracy.

For the $\mathrm{MC}_{\text {eval }}$ case with a $\bar{\sigma}_{\text {min }}$ of $1 \%$, broadly speaking, mean gains are lower because initial GPR errors are lower. They go up to 3\%, allowing to reduce mean GPR errors from maximum $5 \%$ down to less than $2 \%$. However, mean errors are mainly between $0 \%$ and $1 \%$. For the same reason as previously mentioned, a few negative gains of small amplitude are observed.

For a $\bar{\sigma}_{\min }$ of $2 \%$, results similar to the $\mathrm{MC}_{\text {ref }}$ case can be observed, except for the standard deviations (see Appendix C) which are sometimes higher but then correspond to high gains. It seems that, in both cases, using the method for a higher $\bar{\sigma}_{\min }$ still enables a strong reduction of GPR errors but does not guarantee such a good GPR accuracy.

Our results can also be interpreted in terms of computation time. To this end, let us imagine that a noise level as low as $0.3 \%$ is required to consider obtained time gain. For the 10 cases considered, the use of the adapted $\gamma$-index enabled to reduce the Monte Carlo computation time by an approximate factor between 4 and 34 for a $\bar{\sigma}_{\min }$ of $1 \%$, depending on the patient. This means our method was at least 3 times faster than the usual $\gamma$-index. For a $\bar{\sigma}_{\min }$ of $2 \%$, the factor of gain in computation time reached values between 5.2 and 157. For example, the MC simulation for the case Lung 1 required 80 minutes to reach an uncertainty as small as $0.3 \%$, but only 7 and 1.8 minutes respectively to attain $1 \%$ and $2 \%$ of noise. The duration of a single $\gamma$-test remains below 1 minute, depending on the patient and the resolution of the reference dose.

Based on this first part of the discussion, some recommendations can now be made on how to perform appropriate $\gamma$-tests and interpret them correctly when one of the compared dose maps comes from a Monte Carlo calculation.

We first would like to emphasize how important it is to mention how exactly the $\gamma$-test is done when reporting results. Information such as the spatial resolution of compared doses, noise level of the MC dose, normalization factor, and status of each dose (reference or evaluated) should always be mentioned when reporting $\gamma$-evaluations in the litterature.

Then, we believe that when comparing a MC dose to an analytical one, the MC dose should 
preferably be set as reference. Several reasons support this statement. First, the GPR is in that case always underestimated, while for the $\mathrm{MC}_{\text {eval }}$ case it is usually overestimated. It is thus safer, especially in a clinical context, to prefer the $\mathrm{MC}_{\mathrm{ref}}$ option. This is also a good choice to reduce the need of a noise correction method: if the computed GPR is already high enough, no need to worry about its true value. Secondly, the spatial resolution of the evaluated dose strongly affects the GPR and interpolation of the dose distribution is therefore needed. However, applying upsampling to a noisy dose can also lead to biased passing rates, as shown in Section 3.1 and Appendix B. Finally, the MC tool is supposed to provide doses more accurately than analytical algorithms, and it thus makes sense to set MC doses as the references.

When comparing MC doses to measurements, however, our last argument does not hold anymore. Moreover, when setting the MC dose as reference to compare it to a 2D measurement, the search performed by the $\gamma$-test becomes 2D and not 3D anymore, as explained in Section 2.5. A way around this could be to use the $\mathrm{MC}$ dose as evaluated distribution while adapting the GPR passing treshold or using a correction method such as the one proposed here.

Another recommendation would be to impose a lower noise level when computing a local $\gamma$-index, which is more sensitive to noise than a global one. Based on our results, an uncertainty of $1 \%$ seems quite reasonable.

Finally, for the spatial resolution of the evaluated dose, recommendations from AAPM TG-218 should be followed, ${ }^{31}$ i.e., the voxel size in the evaluated dose should remain below $1 / 3$ of the DTA criterion.

This being said, the general framework of the method proposed here could, in our opinion, lead to a good compromise between accuracy and computation time for a QA process involving a $\gamma$-index evaluation based on Monte Carlo dose distributions. However, there might still be some work to be done in order to enable an efficient use of the method. For instance, in order to avoid negative gains occurring when no noise correction is needed, we could imagine to compute beforehand two GPR for two different levels of noise (such as 1\% and 2\% or $2 \%$ and $3 \%$ ) and to deduce from their difference if the adapted $\gamma$-index should be used or not. Likewise, it could be good to explore a way to reduce the variability observed when $\bar{\sigma}_{\text {min }}=2 \%$ (in particular for the $\mathrm{MC}_{\text {eval }}$ case); an option might be to look for another fitting model having fewer parameters or approximating better the shape of GPR curves as a function 
of noise. This could be the scope of further investigation. Another limitation of our method is the case in which we would compare two noisy doses; this is currently not possible since there is no certitude about the impact of noise produced on GPR. A full study should hence be performed to first investigate noise impact then adapt the correction method if needed. Finally, the proposed method was here only tested on PBS proton plans and it might be good to verify if it still applies to other treatment modalities such as conventional radiotherapy. Nonetheless, we believe it should be the case as the shape of the passing rate evolution curve with the statistical MC uncertainty should be independent of the radiation type.

\section{Conclusions}

The dose comparison workflow should be adapted when it involves Monte Carlo doses. Noise impacts $\gamma$ passing rates and worsens the impact of other factors affecting the $\gamma$-index method.

We proposed here an adapted use of the usual $\gamma$-index method for Monte Carlo dose comparison. The idea is to predict the $\gamma$ passing rate for an almost noiseless MC dose based on several passing rates computed for $\mathrm{MC}$ doses having various high levels of noise, with an aim at accuracy gain. This allowed us to reduce the Monte Carlo computation time that would be required to reach a noise-free GPR.

\section{Aknowledgements}

Marie Cohilis is supported by the Télévie Grant from the Belgian "Fonds National pour la Recherche Scientifique" F.R.S-FNRS (Grant No. 7450517F). Kevin Souris is supported by a research grant from Ion Beam Application (IBA s.a., Louvain-la-Neuve, Belgium). John A. Lee is a Research Associate with the Belgian fund of scientific research (F.R.S.-FNRS).

\section{References}

${ }^{1}$ C. H. Clark, A. M. Bidmead, C. D. Mubata, K. J. Harrington, and C. M. Nutting, Intensitymodulated radiotherapy improves target coverage, spinal cord sparing and allows dose escalation in patients with locally advanced cancer of the larynx, Radiother. Oncol. 70, 189-198 (2004). 
${ }^{2}$ X. Zhang, Y. Li, X. Pan, L. Xiaoqiang, R. Mohan, R. Komaki, J. D. Cox, and J. Y. Chang, Intensity-Modulated Proton Therapy Reduces the Dose to Normal Tissue Compared With Intensity-Modulated Radiation Therapy or Passive Scattering Proton Therapy and Enables Individualized Radical Radiotherapy for Extensive Stage IIIB Non-Small-Cell Lung Cancer: A Virtual Clinical Study, Int. J. Radiat. Oncol. Biol. Phys. 77, 357-366 (2010).

${ }^{3}$ G. Ezzell, J. Galvin, D. Low, J. Palta, I. Rosen, M. Sharpe, P. Xia, Y. Xiao, L. Xing, and C. $\mathrm{Yu}$, Guidance document on delivery, treatment planning, and clinical implementation of IMRT: Report of the IMRT subcommittee of the AAPM radiation therapy committee, Med. Phys. 30, 2089-2115 (2003).

${ }^{4}$ D. A. Low, S. Mutic, J. F. Dempsey, R. L. Gerber, W. R. Bosh, C. A. Perez, and J. A. Purdy, Quantitative dosimetric verification of an IMRT planning and delivery system, Radiother. Oncol. 49, 305-316 (1998).

${ }^{5}$ A. Van Esch, J. Bohsung, P. Sorvari, M. Tenhunen, M. Paiusco, M. Iori, P. Engtröm, H. Nyström, and D. P. Huyskens, Acceptance tests and quality control (QC) procedures for the clinical implementation of intensity modulated radiotherapy (IMRT) using inverse planning and the sliding window technique: experience from five radiotherapy departments, Radiother. Oncol. 65, 53-70 (2002).

${ }^{6}$ G. Meier, R. Besson, A. Nanz, S. Safai, and A. Lomax, Independent dose calculations for commissioning, quality assurance and dose reconstruction of PBS proton therapy, Phys. Med. Biol. 60, 2819-2836 (2015).

${ }^{7}$ J. Van Dyk, R. B. Barnett, J. E. Cygler, and P. C. Shragge, Commissioning and quality assurance of treatment planning computers, Int. J. Radiat. Oncol. Biol. Phys. 26, 261-273 (1993).

${ }^{8}$ A. S. Shiu, S. Tung, K. R. Hogström, J. W. Wong, R. L. Gerber, W. B. Harms, J. A. Purdy, R. K. Ten Haken, D. L. McShan, and B. A. Fraass, Verification data for electron beam dose algorithms, Med. Phys. 19, 623-636 (1992).

${ }^{9}$ W. B. Harms, D. A. Low, J. W. Wong, and J. A. Purdy, A software tool for the quantitative evaluation of 3D dose calculation algorithms, Med. Phys. 25, 1830-1836 (1998). 
${ }^{10}$ D. A. Low, W. B. Harms, S. Mutic, and J. A. Purdy, A technique for the quantitative evaluation of dose distributions, Med. Phys. 25, 656-661 (1998).

${ }^{11}$ N. Agazaryan, T. D. Solberg, and J. J. DeMarco, Patient specific quality assurance for the delivery of intensity modulated radiotherapy, J. Appl. Clin. Med. Phys. 4, 40-50 (2003).

${ }^{12}$ M. Wendling, L. J. Zijp, L. N. McDermott, E. J. Smit, J. J. Sonke, B. J. Mijnheer, and M. V. Herk, A fast algorithm for gamma evaluation in 3D, Med. Phys. 34, 1647-1654 (2007).

${ }^{13}$ D. A. Low and J. F. Dempsey, Evaluation of the gamma dose distribution comparison method, Med. Phys. 30, 2455-2464 (2003).

${ }^{14}$ D. A. Low, Gamma Dose Distribution Evaluation Tool, J. Phys.: Conf. Ser. 250, 349-359 (2010).

15 T. Depuydt, A. Van Esch, and D. P. Huyskens, A quantitative evaluation of IMRT dose distributions: refinement and clinical assessment of the gamma evaluation, Radiother. Oncol. 62, 309-319 (2002).

${ }^{16}$ S. B. Jiang, G. C. Sharp, T. Neicu, R. I. Berbeco, S. Flampouri, and T. Bortfeld, On dose distribution comparison, Phys. Med. Biol. 51, 759-776 (2006).

17 J. Y. Huang, K. B. Pulliam, E. M. McKenzie, D. S. Followill, and S. F. Kry, Effects of spatial resolution and noise on gamma analysis for IMRT QA, J. Appl. Clin. Med. Phys. 15, 93-104 (2014).

${ }^{18}$ Y. J. Graves, X. Jia, and S. B. Jiang, Effect of statistical fluctuation in Monte Carlo based photon beam dose calculation on gamma index evaluation, Phys. Med. Biol. 58, 1839-1853 (2013).

${ }^{19}$ U. Jelen and M. Alber, A finite size pencil beam algorithm for IMRT dose optimization: density corrections, Phys. Med. Biol. 52, 617-633 (2007).

${ }^{20}$ S. Hissoiny, B. Ozell, H. Bouchard, and P. Després, GPUMCD: a new GPU-oriented Monte Carlo dose calculation platform, Med. Phys. 38, 754-764 (2011). 
${ }^{21}$ S. Huang, K. Souris, S. Li, M. Kang, A. M. Barragan Montero, J. Guillaume, A. Lin, E. Garver, P. Ainsley, Christopher Taylor, Y. Xiao, and L. Lin, Validation and application of a fast Monte Carlo algorithm for assessing the clinical impact of approximations in analytical dose calculations for pencil beam scanning proton therapy, Med. Phys. 45, 5631-5642 (2018).

${ }^{22}$ T. R. Dhakal and P. Yepes, A symmetric probabilistic gamma-index for Monte Carlo dose comparisons, Phys. Med. Biol. 59, N153-N161 (2014).

${ }^{23}$ G. A. Ezzell, J. W. Burmeister, N. Dogan, T. LoSasso, J. G. Mechalakos, D. Mihailidis, A. Molineu, J. R. Palta, C. R. Ramsey, B. J. Salter, J. Shi, N. Xia, P. Yue, and Y. Xiao, IMRT commissioning: multiple institution planning and dosimetry comparisons, a report from AAPM Task Group 119, Med. Phys. 36, 5359-5373 (2009).

${ }^{24}$ K. Souris, J. A. Lee, and E. Sterpin, Fast multipurpose Monte Carlo simulation for proton therapy using multi- and many-core CPU architectures, Med. Phys. 43, 1700-1712 (2016).

${ }^{25}$ S. Huang, M. Kang, K. Souris, C. Ainsley, T. D. Solberg, J. E. McDonough, C. B. Simone II, and L. Lin, Validation and clinical implementation of an accurate Monte Carlo code for pencil beam scanning proton therapy, J. Appl. Clin. Med. Phys. 19, 558-572 (2018).

${ }^{26} \mathrm{P}$. Andreo, Monte Carlo techniques in medical radiation physics, Phys. Med. Biol. 36, 861-920 (1991).

${ }^{27}$ J. Seco and M. Fragoso, Patient dose calculation, pages 111-124 (2013).

${ }^{28}$ OpenReggui, https://openreggui.org/.

${ }^{29}$ M. Chen, W. Lu, Q. Chen, K. Ruchala, and G. Olivera, Efficient gamma index calculation using fast Euclidean distance transform, Phys. Med. Biol. 54, 2037-2047 (2009).

${ }^{30}$ M. Fippel, Basics of Monte Carlo simulations, pages 17-28 (2013).

${ }^{31}$ M. Miften, A. Olch, D. Mihailidis, J. Moran, T. Pwlicki, A. Molineu, H. Li, K. Wijesooriya, J. Shi, P. Xia, N. Papanikolaou, and D. Low, Tolerance limits and methodologies for IMRT measurement-based verification QA: Recommendations of AAPM Task Group No. 218, Med. Phys. 45, e53-e83 (2018).

${ }^{32}$ C. E. Bonferroni, Teoria statistica delle classi e calcolo delle probabilità, Pubblicazioni del R. Istituto superiore di scienze economiche e commerciali di Firenze 8, 3-62 (1936). 
${ }^{33}$ E. W. Ng and M. Geller, A table of integrals of the error functions, J. Res. Natl. Bur. Stand., Sec. B: Math. Sci. 73B, 1-20 (1969).

\section{Appendix A}

We derive here the upper bound of the GPR given in Eq. (3) and try to show that the model is a good option for our fitting function.

We would like to express the GPR as a function of the mean standard deviation of the MC dose map, noted $\bar{\sigma}$. To do so, we need to derive the mathematical expectation of the GPR. Let's note $m$ the number of voxels in the reference dose (or a subset of it), $n$ the number of voxels in the evaluation dose (or a subset of it), $D$ the reference dose and $\tilde{D}$ the evaluation dose. We will note $r$ or $\tilde{r}$ the spatial coordinates, 3D or 2D, referring to the domain of the reference and evaluation doses respectively.

By definition of the expectation, we have that

$$
\begin{aligned}
G P R & =E\left(\frac{100}{m} \sum_{j=1}^{m} \mathbb{I}_{\gamma_{j} \leq 1}\right) \\
& =\frac{100}{m} \sum_{j=1}^{m} E\left(\mathbb{I}_{\gamma_{j} \leq 1}\right) \\
& =\frac{100}{m} \sum_{j=1}^{m} P\left(\gamma_{j} \leq 1\right),
\end{aligned}
$$

where $\mathbb{I}_{\gamma_{j} \leq 1}$ is the characteristic function defined by

$$
\mathbb{1}_{\gamma_{j} \leq 1}= \begin{cases}1 & \text { if } \gamma_{j} \leq 1 \\ 0 & \text { otherwise }\end{cases}
$$

Developing the gamma value gives us

$$
G P R=\frac{100^{2}}{m} \sum_{j=1}^{m} P\left(\min _{i} \sqrt{\frac{\left(\tilde{r}_{i}-r_{j}\right)^{2}}{\Delta d^{2}}+\frac{100^{2}}{\Delta D^{2}}\left(\frac{\tilde{D}_{i}-D_{j}}{D_{j}}\right)^{2}} \leq 1\right)
$$

and, by Boole's inequality ${ }^{32}$ we get the bound 


$$
\begin{aligned}
G P R & \leq \frac{100}{m} \sum_{j=1}^{m} \sum_{i \in \tilde{N}_{j}} P\left(\frac{100^{2}}{\Delta D^{2}}\left(\frac{\tilde{D}_{i}-D_{j}}{D_{j}}\right)^{2} \leq 1-\frac{\left(\tilde{r}_{i}-r_{j}\right)^{2}}{\Delta d^{2}}\right) \\
& =\frac{100}{m} \sum_{j=1}^{m} \sum_{i \in \tilde{N}_{j}} P\left(\left|1-\frac{\tilde{D}_{i}}{D_{j}}\right| \leq \frac{\Delta D}{100} \sqrt{1-\frac{\left(\tilde{r}_{i}-r_{j}\right)^{2}}{\Delta d^{2}}}\right) \\
& =\frac{100^{2}}{m} \sum_{j=1}^{m} \sum_{i \in \tilde{N}_{j}} P\left(\frac{-\Delta D}{100} \sqrt{1-\frac{\left(\tilde{r}_{i}-r_{j}\right)^{2}}{\Delta d^{2}}}-1 \leq-\frac{\tilde{D}_{i}}{D_{j}} \leq \frac{\Delta D}{100} \sqrt{1-\frac{\left(\tilde{r}_{i}-r_{j}\right)^{2}}{\Delta d^{2}}}-1\right)
\end{aligned}
$$

653

654

where $\tilde{N}_{j}$ is the subset of voxels indices $i$ in the evaluation dose such as

$$
\frac{\left(\tilde{r}_{i}-r_{j}\right)^{2}}{\Delta d^{2}} \leq 1
$$

Here we have to distinguish $\mathrm{MC}_{\mathrm{ref}}$ and $\mathrm{MC}_{\text {eval }}$ cases:

- $M C$ dose is the reference dose:

Assuming hence that $D_{j}$ are random variables $\left(D_{j} \sim N\left(\mu_{j}, \sigma_{j}^{2}\right)\right)$, we get

$$
\begin{aligned}
G P R \leq & \frac{100}{m} \sum_{j=1}^{m} \sum_{i \in \tilde{N}_{j}}\left[P\left(-\frac{\tilde{D}_{i}}{D_{j}} \leq \frac{\Delta D}{100} \sqrt{1-\frac{\left(\tilde{r}_{i}-r_{j}\right)^{2}}{\Delta d^{2}}}-1\right)\right. \\
& -P\left(-\frac{\tilde{D}_{i}}{D_{j}} \leq \frac{-\Delta D}{100} \sqrt{\left.\left.1-\frac{\left(\tilde{r}_{i}-r_{j}\right)^{2}}{\Delta d^{2}}-1\right)\right]}\right. \\
= & \frac{100}{m} \sum_{j=1}^{m} \sum_{i \in \tilde{N}_{j}}\left[P\left(D_{j} \leq \frac{-\tilde{D}_{i}}{\frac{\Delta D}{100} \sqrt{1-\frac{\left(\tilde{r}_{i}-r_{j}\right)^{2}}{\Delta d^{2}}}-1}\right)\right. \\
& -P\left(D_{j} \leq \frac{-\tilde{D}_{i}}{\left.\left.\frac{-\Delta D}{100} \sqrt{1-\frac{\left(\tilde{r}_{i}-r_{j}\right)^{2}}{\Delta d^{2}}-1}\right)\right]}\right. \\
= & \frac{100}{2 m} \sum_{j=1}^{m} \sum_{i \in \tilde{N}_{j}}\left[\operatorname{erf}\left(\frac{P_{i j}-\mu_{j}}{\sqrt{2} \sigma_{j}}\right)-\operatorname{erf}\left(\frac{Q_{i j}-\mu_{j}}{\sqrt{2} \sigma_{j}}\right)\right],
\end{aligned}
$$

where $\operatorname{erf}(\cdot)$ represents the error function, $P_{i j}=\frac{-\tilde{D}_{i}}{\frac{\Delta D}{100} \sqrt{1-\frac{\left(\tilde{r}_{i}-r_{j}\right)^{2}}{\Delta d^{2}}-1}}$ and $Q_{i j}=\frac{-\tilde{D}_{i}}{\frac{-\Delta D}{100} \sqrt{1-\frac{\left(\tilde{r}_{i}-r_{j}\right)^{2}}{\Delta d^{2}}}-1}$ 
- $M C$ dose is the evaluated dose:

Let's assume now that $\forall i, \tilde{D}_{i} \sim N\left(\tilde{\mu}_{i}, \tilde{\sigma}_{i}^{2}\right)$. Similarly to the previous case, we obtain

$$
\begin{aligned}
G P R \leq & \frac{100}{m} \sum_{j=1}^{m} \sum_{i \in \tilde{N}_{j}}\left[P\left(\frac{\tilde{D}_{i}}{D_{j}} \leq \frac{\Delta D}{100} \sqrt{1-\frac{\left(\tilde{r}_{i}-r_{j}\right)^{2}}{\Delta d^{2}}}+1\right)\right. \\
& -P\left(\frac{\tilde{D}_{i}}{D_{j}} \leq \frac{-\Delta D}{100} \sqrt{\left.\left.1-\frac{\left(\tilde{r}_{i}-r_{j}\right)^{2}}{\Delta d^{2}}+1\right)\right] .}\right. \\
G P R \leq & \frac{100}{m} \sum_{j=1}^{m} \sum_{i \in \tilde{N}_{j}}\left[P \left(\tilde{D}_{i} \leq D_{j}\left(\frac{\Delta D}{100} \sqrt{\left.\left.1-\frac{\left(\tilde{r}_{i}-r_{j}\right)^{2}}{\Delta d^{2}}+1\right)\right)}\right.\right.\right. \\
& -P\left(\tilde{D}_{i} \leq D_{j}\left(\frac{-\Delta D}{100} \sqrt{\left.1-\frac{\left(\tilde{r}_{i}-r_{j}\right)^{2}}{\Delta d^{2}}+1\right)}\right)\right] \\
= & \frac{100}{2 m} \sum_{j=1}^{m} \sum_{i \in \tilde{N}_{j}}\left[\operatorname{erf}\left(\frac{P_{i j}-\tilde{\mu}_{i}}{\sqrt{2} \tilde{\sigma}_{i}}\right)-\operatorname{erf}\left(\frac{Q_{i j}-\tilde{\mu}_{i}}{\sqrt{2} \tilde{\sigma}_{i}}\right)\right],
\end{aligned}
$$

$$
\text { with } P_{i j}=D_{j}\left(\frac{\Delta D}{100} \sqrt{1-\frac{\left(\tilde{r}_{i}-r_{j}\right)^{2}}{\Delta d^{2}}}+1\right) \text { and } Q_{i j}=D_{j}\left(\frac{-\Delta D}{100} \sqrt{1-\frac{\left(\tilde{r}_{i}-r_{j}\right)^{2}}{\Delta d^{2}}}+1\right) \text {. }
$$

This means that the GPR can be bound by a sum of many error functions, $m$ being quite large, for both $\mathrm{MC}_{\mathrm{ref}}$ and $\mathrm{MC}_{\text {eval }}$ cases.

Next, what we would like to obtain is a GPR expression as a function of the mean uncertainty of the MC dose, $\bar{\sigma}$. In order to do so, we will assume that each Monte Carlo dose voxel has an uncertainty being proportional to $\bar{\sigma}$, meaning that for each voxel, it exists a factor $\beta_{j}$ for the $\mathrm{MC}_{\text {ref }}$ case or $\tilde{\beta}_{i}$ for the $\mathrm{MC}_{\text {eval }}$ case, such as

$$
\sigma_{i}=\frac{1}{\tilde{\beta}_{i}} \bar{\sigma}
$$

or

$$
\sigma_{j}=\frac{1}{\beta_{j}} \bar{\sigma}
$$

regardless of the noise level. As it is not exactly the case, we are here introducing a small approximation in our model. Replacing (24) and (25) into (20) and (23) respectively, we get

$$
G P R \leq \frac{100}{2 m} \sum_{j=1}^{m} \sum_{i \in \tilde{N}_{j}}\left[\operatorname{erf}\left(\frac{\beta_{j}\left(P_{i j}-\mu_{j}\right)}{\sqrt{2} \bar{\sigma}}\right)-\operatorname{erf}\left(\frac{\beta_{j}\left(Q_{i j}-\mu_{j}\right)}{\sqrt{2} \bar{\sigma}}\right)\right]
$$


for the $\mathrm{MC}_{\mathrm{ref}}$ case, and

$$
G P R \leq \frac{100}{2 m} \sum_{j=1}^{m} \sum_{i \in \tilde{N}_{j}}\left[\operatorname{erf}\left(\frac{\tilde{\beta}_{i}\left(P_{i j}-\tilde{\mu}_{i}\right)}{\sqrt{2} \bar{\sigma}}\right)-\operatorname{erf}\left(\frac{\tilde{\beta}_{i}\left(Q_{i j}-\tilde{\mu}_{i}\right)}{\sqrt{2} \bar{\sigma}}\right)\right]
$$

for the $\mathrm{MC}_{\text {eval }}$ case.

As we see it, two options are open to us from here.

We can choose to use a parametrized sum of a few error functions as our fitting model, truncating the sum that we have here. Another option is to approximate this sum with an integral, being aware that this will introduce another important approximation in the model. We decided to go for the second option here. For both $\mathrm{MC}_{\mathrm{ref}}$ and $\mathrm{MC}_{\text {eval }}$ cases, our upper bound can be rewritten

$$
G P R \leq \frac{100}{2 m} \sum_{k=1}^{S}\left[\operatorname{erf}\left(\frac{A_{k}}{\bar{\sigma}}\right)-\operatorname{erf}\left(\frac{B_{k}}{\bar{\sigma}}\right)\right],
$$

where the $\left\{A_{k}\right\}_{k=1, \ldots, S}$ and $\left\{B_{k}\right\}_{k=1, \ldots, S}$ would be defined by

$$
\left\{\begin{array}{l}
A_{k}:=\beta_{j}\left(P_{i j}-\mu_{j}\right) \\
B_{k}:=\beta_{j}\left(Q_{i j}-\mu_{j}\right)
\end{array}\right.
$$

for the $\mathrm{MC}_{\mathrm{ref}}$ case, and by

$$
\left\{\begin{array}{l}
A_{k}:=\tilde{\beta}_{i}\left(P_{i j}-\tilde{\mu}_{i}\right) \\
B_{k}:=\tilde{\beta}_{i}\left(Q_{i j}-\tilde{\mu}_{i}\right)
\end{array}\right.
$$

for the $\mathrm{MC}_{\text {eval }}$ case, considering given $i$ and $j$ so that the sum over $k$ would replace both sums over $i$ and $j$.

We can then use the integral definition for any function $f$, given by

$$
\int_{a}^{b} f(x) d x=\lim _{j} \sum_{j} \sum_{i=1}^{Q} f\left(c_{i}\right) \Delta x_{i}
$$

where $\Delta x_{i}=x_{i}-x_{i-1} c_{0}=a, c_{Q}=b$ and $c_{i} \in\left[x_{i-1}, x_{i}\right]$. If we further make the hypothesis of a uniform mesh, meaning that $\forall i \Delta x_{i}=\frac{b-a}{Q}:=\Delta x$, then we get

$$
\int_{a}^{b} f(x) d x=\lim _{\Delta x \rightarrow 0} \sum_{i=1}^{Q} f\left(c_{i}\right) \Delta x
$$

and for $\Delta x$ small,

$$
\frac{1}{\Delta x} \int_{a}^{b} f(x) d x \simeq \sum_{i=1}^{Q} f\left(c_{i}\right)
$$


Equation (33) can be used in our case to approximate a sum of error functions by an integral:

$$
\left\{\begin{aligned}
\sum_{k=1}^{S} \operatorname{erf}\left(\frac{A_{k}}{\bar{\sigma}}\right) \simeq \frac{1}{\Delta A} \int_{\min _{k} A_{k}}^{\max A_{k}} \operatorname{erf}\left(\frac{A}{\bar{\sigma}}\right) d A \\
\sum_{k=1}^{S} \operatorname{erf}\left(\frac{B_{k}}{\bar{\sigma}}\right) \simeq \frac{1}{\Delta B} \int_{\min _{k} B_{k}}^{\max _{k} B_{k}} \operatorname{erf}\left(\frac{B}{\bar{\sigma}}\right) d B .
\end{aligned}\right.
$$

Replacing this in (28) and developing the integral of the error function given by $\mathrm{Ng}$ et al., ${ }^{33}$ we finally obtain

$$
\begin{aligned}
G P R \leq & \frac{100}{2 m}\left[\frac { 1 } { \Delta A } \left(M_{A} \operatorname{erf}\left(\frac{M_{A}}{\bar{\sigma}}\right)+\frac{\bar{\sigma}}{\sqrt{\pi}} \mathrm{e}^{-\left(\frac{M_{A}}{\bar{\sigma}}\right)^{2}}-m_{A} \operatorname{erf}\left(\frac{m_{A}}{\bar{\sigma}}\right)-\frac{\bar{\sigma}}{\sqrt{\pi}} \mathrm{e}^{\left.-\left(\frac{m_{A}}{\bar{\sigma}}\right)^{2}\right)}\right.\right. \\
& -\frac{1}{\Delta B}\left(M_{B} \operatorname{erf}\left(\frac{M_{B}}{\bar{\sigma}}\right)+\frac{\bar{\sigma}}{\sqrt{\pi}} \mathrm{e}^{-\left(\frac{M_{B}}{\bar{\sigma}}\right)^{2}}-m_{B} \operatorname{erf}\left(\frac{m_{B}}{\bar{\sigma}}\right)-\frac{\bar{\sigma}}{\sqrt{\pi}} \mathrm{e}^{\left.\left.-\left(\frac{m_{B}}{\bar{\sigma}}\right)^{2}\right)\right],}\right.
\end{aligned}
$$

with $m_{A}=\min _{k} A_{k}, M_{A}=\max _{k} A_{k}, m_{B}=\min _{k} B_{k}$ and $M_{B}=\max _{k} B_{k}$. This leads to the parametrized equation given in (3).

Although this is probably not a tight bound on the GPR due to the approximations made, this function was found suitable for fitting both $\mathrm{MC}_{\mathrm{ref}}$ and $\mathrm{MC}_{\text {eval }}$ cases since it provided a very good fit for all cases when using every available point as fitting point. Figure 12 illustrates this for the $2 \% / 2 \mathrm{~mm} H \& N 1$ case.

Note that it can be shown that the same bound is valid for a global $\gamma$-index as well.
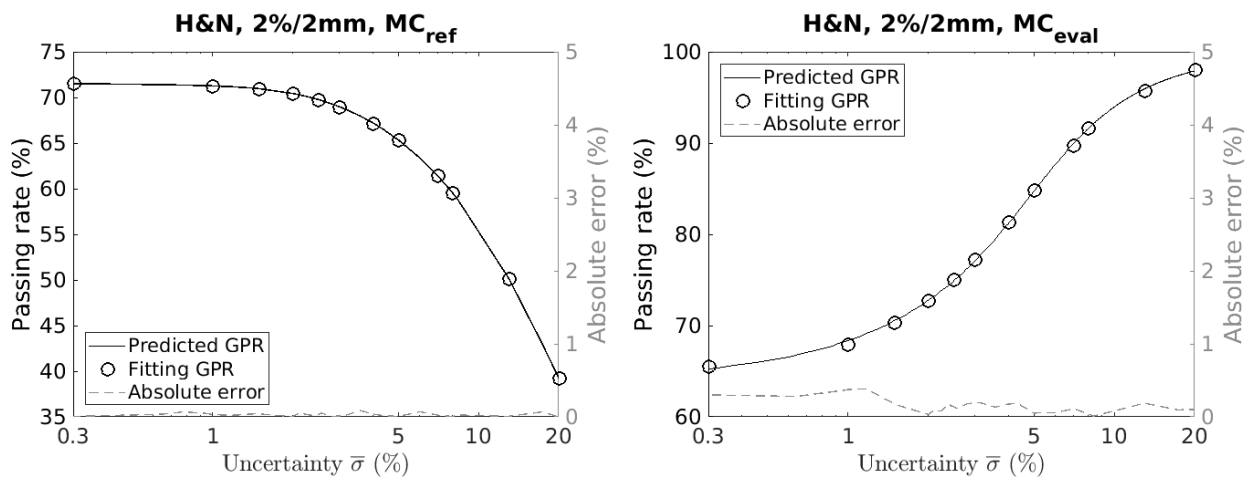

Figure 12: Illustration of the possibility of a good fitting with the chosen model of Equation (3) : resulting fit and corresponding absolute error for (a) $\mathrm{H} \& \mathrm{~N} 1 \mathrm{MC}_{\text {ref }}$ case, $2 \% / 2 \mathrm{~mm}$; (b) $\mathrm{H} \& \mathrm{~N} 1 \mathrm{MC}_{\text {eval }}$ case, $2 \% / 2 \mathrm{~mm}$. The circles show the points used for fitting, the plain curve shows the predicted passing rate and the dashed line the absolute error. 


\section{Appendix B}

Table 1 shows for five different cases and four different criteria the error committed on the GPR at various statistical uncertainties, when no noise correction is made.

To analyze these results, we study first the $\mathrm{MC}_{\text {ref }}$ case, then the $\mathrm{MC}_{\text {eval }}$ case. Several criteria susceptible to affect GPR are considered: the DTA criterion, the volume of the compared regions of interest (ROI) and the true (noise-free) passing rate.

\section{MC dose set as reference dose:}

\section{- DTA criterion:}

By looking at the magnitude of errors for the $\mathrm{MC}_{\text {ref }}$ case, we observe that they are spread in a wide range of negative values, depending on the criterion and the patient. For each patient, the error magnitude seems to increase for a decreasing

\begin{tabular}{|c|c|c|c|c|c|c|c|c|c|}
\hline \multirow[t]{2}{*}{ Case } & & \multicolumn{8}{|c|}{ Error on the GPR for a given $\bar{\sigma}(\%)$} \\
\hline & & \multirow{2}{*}{$\begin{array}{c}\mathbf{G P R}_{\overline{\boldsymbol{\sigma}}_{0}} \\
84.52\end{array}$} & \multicolumn{3}{|c|}{$\bar{\sigma}=1 \% \quad \bar{\sigma}=2 \% \quad \bar{\sigma}=3 \%$} & $\mathbf{G P R}_{\bar{\sigma}_{0}}$ & \multicolumn{2}{|c|}{$\begin{array}{c}\bar{\sigma}= \\
\left(\mathrm{MC}_{\text {eval }}\right)\end{array}$} & $\bar{\sigma}=3 \%$ \\
\hline \multirow[t]{4}{*}{ Prostate } & $2 \% / 2 \mathrm{~mm}$ & & -1.57 & -6.65 & -11.76 & 91.00 & +5.27 & +7.91 & +8.55 \\
\hline & $3 \% / 3 \mathrm{~mm}$ & 94.07 & -1.37 & -5.15 & -9.82 & 98.21 & +1.22 & +1.41 & +1.45 \\
\hline & $4 \% / 4 \mathrm{~mm}$ & 98.13 & -0.97 & -3.64 & -7.39 & 99.60 & +0.08 & +0.11 & +0.16 \\
\hline & $3 \% / 1 \mathrm{~mm}$ & 84.22 & -2.01 & -7.08 & -12.95 & 86.89 & +1.57 & +3.77 & +5.29 \\
\hline \multirow[t]{4}{*}{ Lung } & $2 \% / 2 \mathrm{~mm}$ & 89.26 & -5.50 & -14.18 & -21.12 & 89.59 & +1.56 & +3.19 & +4.36 \\
\hline & $3 \% / 3 \mathrm{~mm}$ & 92.69 & -2.38 & -7.84 & -13.23 & 93.17 & +1.06 & +2.45 & +3.29 \\
\hline & $4 \% / 4 \mathrm{~mm}$ & 94.63 & -1.16 & -4.65 & -8.66 & 95.26 & +0.85 & +1.80 & +2.31 \\
\hline & $3 \% / 1 \mathrm{~mm}$ & 83.83 & -8.03 & -19.53 & -28.36 & 83.95 & +0.43 & +0.73 & +0.45 \\
\hline \multirow[t]{4}{*}{ Brain } & $2 \% / 2 \mathrm{~mm}$ & 77.22 & -0.30 & -1.55 & -3.56 & 74.97 & +4.68 & +11.38 & +15.12 \\
\hline & $3 \% / 3 \mathrm{~mm}$ & 90.00 & -0.39 & -1.66 & -3.52 & 86.88 & +3.36 & +6.26 & +7.78 \\
\hline & $4 \% / 4 \mathrm{~mm}$ & 95.05 & -0.23 & -0.98 & -2.22 & 91.68 & +2.07 & +3.68 & +4.62 \\
\hline & $3 \% / 1 \mathrm{~mm}$ & 70.01 & -0.48 & -2.16 & -4.54 & 68.15 & +1.48 & +4.51 & +7.46 \\
\hline \multirow[t]{4}{*}{ Liver } & $2 \% / 2 \mathrm{~mm}$ & 94.11 & -2.87 & -6.08 & -10.40 & 95.40 & +1.44 & +1.96 & +2.43 \\
\hline & $3 \% / 3 \mathrm{~mm}$ & 98.83 & -1.22 & -3.33 & -6.57 & 98.76 & +0.23 & +0.30 & +0.37 \\
\hline & $4 \% / 4 \mathrm{~mm}$ & 99.71 & -0.31 & -1.23 & -3.14 & 99.33 & +0.04 & +0.10 & +0.19 \\
\hline & $3 \% / 1 \mathrm{~mm}$ & 93.27 & -2.62 & -6.19 & -11.79 & 93.45 & -0.31 & -0.92 & -1.45 \\
\hline \multirow[t]{4}{*}{$H E N$} & $2 \% / 2 \mathrm{~mm}$ & 71.53 & -0.24 & -1.10 & -2.55 & 65.46 & +2.52 & +7.29 & +11.80 \\
\hline & $3 \% / 3 \mathrm{~mm}$ & 83.35 & -0.19 & -0.75 & -1.80 & 76.59 & +3.13 & +7.90 & +11.73 \\
\hline & $4 \% / 4 \mathrm{~mm}$ & 90.74 & -0.16 & -0.62 & -1.46 & 83.95 & +3.25 & +7.13 & +9.77 \\
\hline & $3 \% / 1 \mathrm{~mm}$ & 58.81 & -0.37 & -1.58 & -3.49 & 55.15 & +1.08 & +3.63 & +6.43 \\
\hline
\end{tabular}

Table 1: Summary of the errors made on the GPR when using the GPR for $\bar{\sigma}=1 \%, \bar{\sigma}=2 \%$ and $\bar{\sigma}=3 \%$. The reference GPR $\left(\mathrm{GPR}_{\bar{\sigma}_{0}}\right)$ is here the GPR for the smallest uncertainty available for the corresponding patient, i.e. $0.24 \%, 0.27 \%, 0.30 \%, 0.30 \%$ and $0.23 \%$ respectively for prostate, lung, brain, liver and H\&N. The error is defined as $\mathrm{GPR}_{\bar{\sigma}}-\mathrm{GPR}_{\bar{\sigma}_{0}}$. 
DTA. This makes sense since the utility of the DTA criterion is to provide a search radius in order to relax the constraints of a point-by-point dose difference. Thus, the larger the DTA criterion, the bigger the search radius and the least the impact of noise. But for a same DTA criterion, various GPR errors can be observed. For instance, a $3 \% / 1 \mathrm{~mm} \gamma$-test leads to deviations between $-8.03 \%$ and $-0.37 \%$ for a $\bar{\sigma}$ of $1 \%$, i.e. noise impact can be significant or not at all.

- Volume of ROI:

GPR error was drawn as a function of ROI volume, for criteria of $2 \% / 2 \mathrm{~mm}$ and $3 \% / 3 \mathrm{~mm}$, and using $\bar{\sigma}=2 \%$. Figure $13(\mathrm{a})$ shows the results. No dependence seems to be visible.

- True GPR:

Figure 13(b) shows the dependence between true (noise-free) GPR and GPR error obtained for $\bar{\sigma}=2 \%$. Here there might be a light trend of negative correlation: it looks like the higher the true passing rate, the higher the absolute error. However, for one patient, it is not the case. Drawing general conclusions is here complicated.

\section{MC dose set as evaluated dose:}

- DTA criterion:

This time, the dependence of the GPR error to the DTA criterion is not always present. Indeed, for all patients, a criterion of $3 \% / 3 \mathrm{~mm}$ leads to an error lower

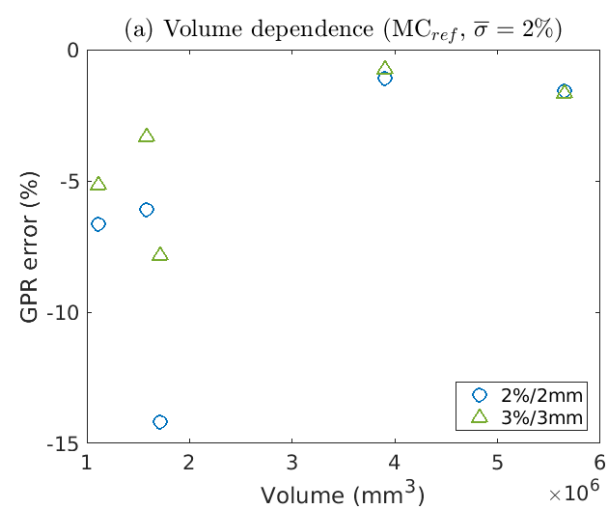

(a)

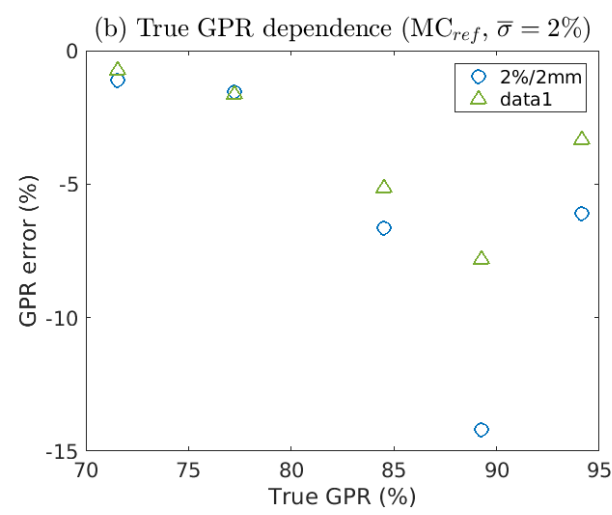

(b)

Figure 13: $\mathrm{MC}_{\text {ref }}$ case with $\bar{\sigma}=2$ : (a) Evaluation of the dependence between GPR error and dose volume processed in the $\gamma$-test; (b) Evaluation of the dependence between GPR error and the true passing rate. 
than for the $2 \% / 2 \mathrm{~mm}$ case. We believe this might be linked to the interpolation of MC doses and, indirectly, to the shape of GPR evolution curves. In Figure 14, for the prostate case, we can see that GPR is first increasing when noise decreases before going down as it should. An extreme example is the liver case (see Table 1), for which the GPR error is actually negative. We believe there are two possible explanations for this phenomenon to occur. First, it might be linked to the small DTA criterion which, at a high noise level, might not be sufficient to compensate noise magnitude. However, this would not justify what happens with the liver case, which has raising GPR until a statistical uncertainty as small as $0.3 \%$. Another hypothesis could be that the interpolation of MC doses in the $\gamma$-test skews the results. Although the impact of resampling was checked previously (see Section 2.3.2), the $3 \% / 1 \mathrm{~mm}$ case was not considered because the used resolution ([0.39, $0.39,0.5] \mathrm{mm}$ ) was not high enough to meet AAPM recommendations. ${ }^{31}$ But if we make the comparison anyway between interpolated and recomputed doses for such a small DTA criterion, keeping in mind that a small resolution effect might be present, we see (Figure 15) that it is indeed the interpolation that causes this behavior: initial doses have a voxel size higher than the DTA criterion and interpolation does not seem to be able to prevent GPR evolution curves from having a behavior similar to the case of a DD criterion only. However, as noise becomes very small, passing rates seem to tend to a same value, which confirms that interpolating noisy doses should be avoided while resampling a smooth dose is acceptable.

- Volume of ROI:

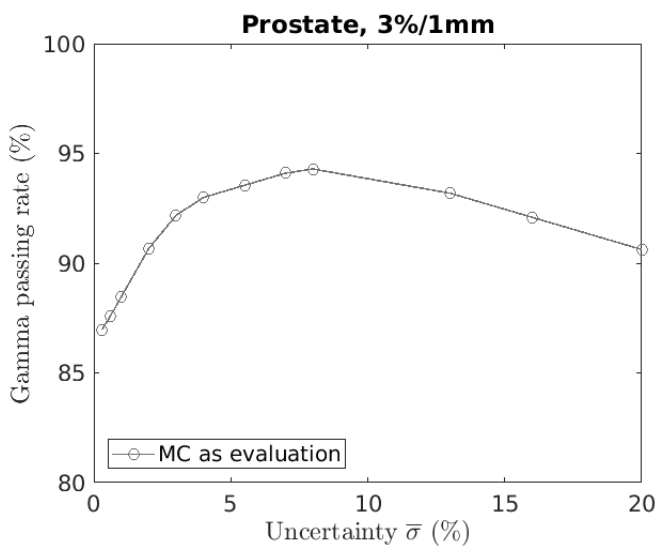

Figure 14: GPR as a function of noise level for the prostate $\mathrm{MC}_{\text {ref }}$ case with a criterion of $3 \% / 1 \mathrm{~mm}$. 


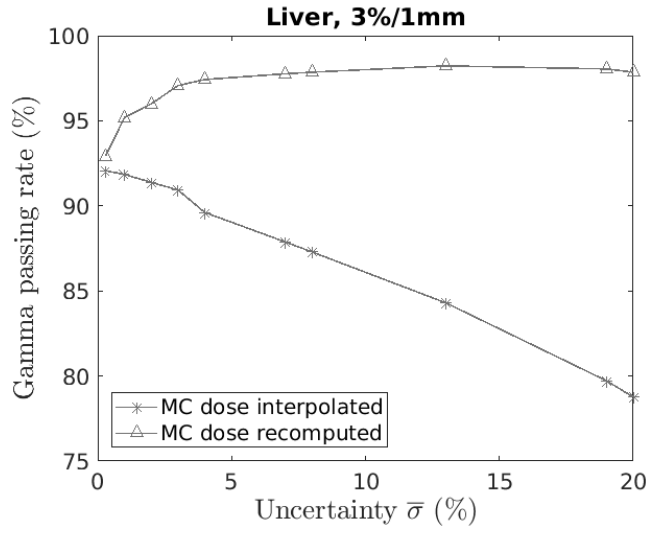

Figure 15: Comparison of interpolated and directly computed $\mathrm{MC}$ evaluated doses in the liver case, for $3 \% / 1 \mathrm{~mm}$ GPR. Resolution of the MC dose is $[0.39,0.39,0.5] \mathrm{mm}$.

Figure 16(a) shows the dependence of GPR error on volume. Again, no dependence on the volume is observed.

- True GPR:

Figure 16(b) shows the dependence of GPR error on the true passing rate. Here there might be a correlation between GPR error and true GPR; a lower true passing rate would lead to a noise impact more consequent. This makes sense as the GPR can not exceed $100 \%$. If a true GPR of $100 \%$ is expected, no overestimation can thus occur. Note that similar (and clearer) trend was noticed by Huang et al., ${ }^{17}$ but for a global $\gamma$-index.
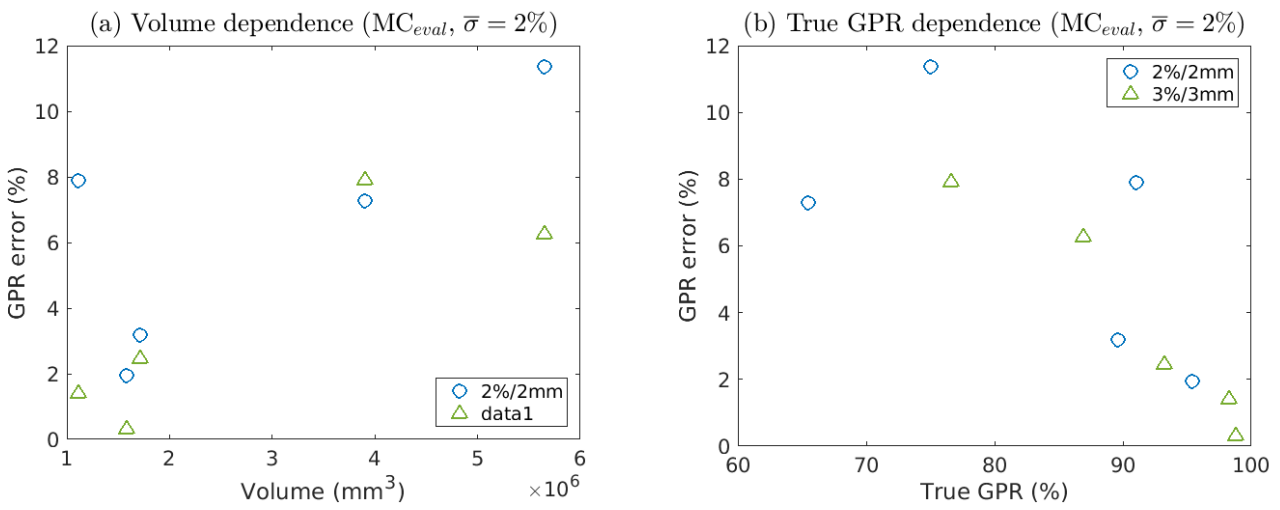

Figure 16: $\mathrm{MC}_{\text {eval }}$ case with $\bar{\sigma}=2$ : (a) Evaluation of the dependence between GPR error and dose volume processed in the $\gamma$-test; (b) Evaluation of the dependence between GPR error and the true passing rate. 


\section{${ }_{770}$ Appendix $\mathrm{C}$}

771 Table 2 summarizes the results obtained for each patient and each $\gamma$-criterion when $\bar{\sigma}_{\min }=1 \%$.

772 The true passing rates for a noise level of $0.3 \%$ are given, along with mean estimated GPR, 773 mean errors, mean gains and standard deviations achieved by the method. These standard 774 deviations are relative to mean errors and gains; indeed, as the optimization process in our 775 method uses randomly selected initial conditions, the method was tested here 60 times to 776 verify its stability and mean values with corresponding standard deviations are reported. Simi777 larly, Table 3 shows the results obtained for each patient and each $\gamma$-criterion when $\bar{\sigma}_{\min }=2 \%$.

Table 4 and Table 5 then propose the same contents as Table 1 and 2 , but for the $\mathrm{MC}_{\text {eval }}$ case. 


\begin{tabular}{|c|c|c|c|c|c|c|}
\hline Case & & $\begin{array}{c}\mathbf{G P R}_{\text {true, } \mathbf{1 \%}} \\
(\%) \\
\end{array}$ & $\begin{array}{c}\mathbf{G P R}_{\text {true, } \mathbf{0 . 3 \%}} \\
(\%)\end{array}$ & $\begin{array}{c}\mathbf{G P R}_{\text {predicted,0.3\% }} \\
(\%)\end{array}$ & $\begin{array}{c}\text { Absolute error } \\
(\%)\end{array}$ & $\begin{array}{c}\text { Gain } \\
(\%) \\
\end{array}$ \\
\hline \multirow[t]{4}{*}{ Prostate } & $2 \% / 2 \mathrm{~mm}$ & 82.95 & 84.44 & 86.39 & $1.96 \pm 0.03$ & $-0.53 \pm 0.03$ \\
\hline & $3 \% / 3 \mathrm{~mm}$ & 92.69 & 93.96 & 93.92 & $0.04 \pm 0.00$ & $1.20 \pm 0.00$ \\
\hline & $4 \% / 4 \mathrm{~mm}$ & 97.16 & 98.10 & 97.79 & $0.30 \pm 0.00$ & $0.63 \pm 0.00$ \\
\hline & $3 \% / 1 \mathrm{~mm}$ & 82.22 & 84.11 & 85.73 & $1.62 \pm 0.00$ & $0.24 \pm 0.00$ \\
\hline \multirow[t]{4}{*}{ Lung 1} & $2 \% / 2 \mathrm{~mm}$ & 83.76 & 89.06 & 89.51 & $0.44 \pm 0.12$ & $4.86 \pm 0.12$ \\
\hline & $3 \% / 3 \mathrm{~mm}$ & 90.31 & 92.63 & 93.53 & $0.90 \pm 0.06$ & $1.43 \pm 0.06$ \\
\hline & $4 \% / 4 \mathrm{~mm}$ & 93.47 & 94.61 & 94.90 & $0.29 \pm 0.00$ & $0.85 \pm 0.00$ \\
\hline & $3 \% / 1 \mathrm{~mm}$ & 75.80 & 83.54 & 82.86 & $0.68 \pm 0.28$ & $7.06 \pm 0.28$ \\
\hline \multirow[t]{4}{*}{ Brain 1} & $2 \% / 2 \mathrm{~mm}$ & 76.92 & 77.22 & 77.76 & $0.54 \pm 0.00$ & $-0.25 \pm 0.00$ \\
\hline & $3 \% / 3 \mathrm{~mm}$ & 89.61 & 90.00 & 90.47 & $0.47 \pm 0.00$ & $-0.08 \pm 0.00$ \\
\hline & $4 \% / 4 \mathrm{~mm}$ & 94.82 & 95.05 & 95.34 & $0.28 \pm 0.00$ & $-0.05 \pm 0.00$ \\
\hline & $3 \% / 1 \mathrm{~mm}$ & 69.53 & 70.01 & 70.65 & $0.64 \pm 0.01$ & $-0.16 \pm 0.01$ \\
\hline \multirow[t]{4}{*}{ Liver } & $2 \% / 2 \mathrm{~mm}$ & 91.24 & 94.11 & 92.25 & $1.86 \pm 0.00$ & $1.01 \pm 0.00$ \\
\hline & $3 \% / 3 \mathrm{~mm}$ & 97.62 & 98.83 & 98.47 & $0.36 \pm 0.00$ & $0.85 \pm 0.00$ \\
\hline & $4 \% / 4 \mathrm{~mm}$ & 99.41 & 99.71 & 99.91 & $0.19 \pm 0.00$ & $0.11 \pm 0.00$ \\
\hline & $3 \% / 1 \mathrm{~mm}$ & 90.66 & 93.27 & 92.18 & $1.10 \pm 0.00$ & $1.52 \pm 0.00$ \\
\hline \multirow[t]{4}{*}{$H E S N 1$} & $2 \% / 2 \mathrm{~mm}$ & 71.29 & 71.52 & 71.65 & $0.14 \pm 0.09$ & $0.07 \pm 0.09$ \\
\hline & $3 \% / 3 \mathrm{~mm}$ & 83.16 & 83.33 & 83.45 & $0.14 \pm 0.01$ & $0.02 \pm 0.01$ \\
\hline & $4 \% / 4 \mathrm{~mm}$ & 90.58 & 90.72 & 90.84 & $0.12 \pm 0.02$ & $0.01 \pm 0.02$ \\
\hline & $3 \% / 1 \mathrm{~mm}$ & 58.45 & 58.78 & 59.00 & $0.23 \pm 0.01$ & $0.08 \pm 0.01$ \\
\hline \multirow{4}{*}{$\begin{array}{l}\text { Lung } 2 \text { - POST } \\
\text { (PSQA) }\end{array}$} & $2 \% / 2 \mathrm{~mm}$ & 95.05 & 96.83 & 97.09 & $0.22 \pm 0.00$ & $1.60 \pm 0.00$ \\
\hline & $3 \% / 3 \mathrm{~mm}$ & 99.49 & 99.78 & 99.93 & $0.15 \pm 0.00$ & $0.14 \pm 0.00$ \\
\hline & $4 \% / 4 \mathrm{~mm}$ & 99.96 & 99.97 & 100.00 & $0.02 \pm 0.00$ & $-0.00 \pm 0.00$ \\
\hline & $3 \% / 1 \mathrm{~mm}$ & 92.32 & 92.93 & 94.44 & $1.49 \pm 0.09$ & $-0.87 \pm 0.09$ \\
\hline \multirow{4}{*}{$\begin{array}{l}\text { Lung } 2-L P O \\
\text { (PSQA) }\end{array}$} & $2 \% / 2 \mathrm{~mm}$ & 96.73 & 97.75 & 98.53 & $0.78 \pm 0.00$ & $0.25 \pm 0.00$ \\
\hline & $3 \% / 3 \mathrm{~mm}$ & 99.48 & 99.51 & 99.93 & $0.42 \pm 0.00$ & $-0.39 \pm 0.00$ \\
\hline & $4 \% / 4 \mathrm{~mm}$ & 99.89 & 99.97 & 99.92 & $0.05 \pm 0.01$ & $0.03 \pm 0.01$ \\
\hline & $3 \% / 1 \mathrm{~mm}$ & 95.56 & 96.26 & 97.22 & $0.95 \pm 0.04$ & $-0.25 \pm 0.04$ \\
\hline \multirow[t]{4}{*}{ Lung 3} & $2 \% / 2 \mathrm{~mm}$ & 86.95 & 90.28 & 91.84 & $1.56 \pm 0.15$ & $1.77 \pm 0.15$ \\
\hline & $3 \% / 3 \mathrm{~mm}$ & 95.19 & 96.45 & 91.84 & $1.56 \pm 0.15$ & $1.77 \pm 0.15$ \\
\hline & $4 \% / 4 \mathrm{~mm}$ & 98.03 & 98.48 & 98.94 & $0.46 \pm 0.00$ & $-0.02 \pm 0.00$ \\
\hline & $3 \% / 1 \mathrm{~mm}$ & 80.72 & 83.96 & 84.13 & $0.17 \pm 0.32$ & $3.07 \pm 0.32$ \\
\hline \multirow[t]{4}{*}{ Brain 2} & $2 \% / 2 \mathrm{~mm}$ & 91.92 & 92.71 & 92.69 & $0.02 \pm 0.00$ & $0.78 \pm 0.00$ \\
\hline & $3 \% / 3 \mathrm{~mm}$ & 97.06 & 97.45 & 97.65 & $0.20 \pm 0.00$ & $0.19 \pm 0.00$ \\
\hline & $4 \% / 4 \mathrm{~mm}$ & 98.86 & 99.06 & 99.38 & $0.32 \pm 0.03$ & $-0.12 \pm 0.03$ \\
\hline & $3 \% / 1 \mathrm{~mm}$ & 83.52 & 84.62 & 84.54 & $0.07 \pm 0.00$ & $1.03 \pm 0.00$ \\
\hline \multirow[t]{4}{*}{$H \& N^{2}$} & $2 \% / 2 \mathrm{~mm}$ & 93.50 & 93.81 & 94.37 & $0.56 \pm 0.02$ & $-0.25 \pm 0.02$ \\
\hline & $3 \% / 3 \mathrm{~mm}$ & 98.16 & 98.25 & 98.56 & $0.32 \pm 0.06$ & $-0.23 \pm 0.06$ \\
\hline & $4 \% / 4 \mathrm{~mm}$ & 99.26 & 99.28 & 99.34 & $0.07 \pm 0.06$ & $-0.05 \pm 0.06$ \\
\hline & $3 \% / 1 \mathrm{~mm}$ & 85.54 & 85.88 & 86.79 & $0.91 \pm 0.00$ & $-0.59 \pm 0.00$ \\
\hline
\end{tabular}

Table 2: Summary of the results for the $\mathrm{MC}_{\text {ref }}$ case when $\bar{\sigma}_{\min }=1 \%$. The true passing rates for $\bar{\sigma}=\bar{\sigma}_{\min }$ and $\bar{\sigma}=0.3 \%$ are shown, along with the predicted passing rate for $\bar{\sigma}=0.3 \%$. The two last columns show the absolute error made by predicting the GPR at $\bar{\sigma}=0.3 \%$ as well as the gain as defined in Eq. (8) (average \pm standard deviation). 


\begin{tabular}{|c|c|c|c|c|c|c|}
\hline Case & & $\begin{array}{c}\mathbf{G P R}_{\text {true }, \mathbf{2} \%} \\
(\%) \\
\end{array}$ & $\begin{array}{c}\mathbf{G P R}_{\text {true, } \mathbf{0 . 3 \%}} \\
(\%)\end{array}$ & $\begin{array}{c}\mathbf{G P R}_{\text {predicted,0.3\% }} \\
(\%)\end{array}$ & $\begin{array}{c}\text { Absolute error } \\
(\%)\end{array}$ & $\begin{array}{c}\text { Gain } \\
(\%)\end{array}$ \\
\hline \multirow[t]{4}{*}{ Prostate } & $2 \% / 2 \mathrm{~mm}$ & 77.86 & 84.44 & 86.20 & $1.76 \pm 0.05$ & $4.81 \pm 0.05$ \\
\hline & $3 \% / 3 \mathrm{~mm}$ & 88.92 & 93.96 & 96.72 & $2.76 \pm 0.05$ & $2.21 \pm 0.05$ \\
\hline & $4 \% / 4 \mathrm{~mm}$ & 94.49 & 98.10 & 99.04 & $0.95 \pm 0.00$ & $2.57 \pm 0.00$ \\
\hline & $3 \% / 1 \mathrm{~mm}$ & 77.14 & 84.11 & 87.19 & $3.08 \pm 0.49$ & $3.84 \pm 0.49$ \\
\hline \multirow[t]{4}{*}{ Lung 1} & $2 \% / 2 \mathrm{~mm}$ & 75.08 & 89.06 & 86.28 & $2.79 \pm 0.28$ & $11.26 \pm 0.28$ \\
\hline & $3 \% / 3 \mathrm{~mm}$ & 84.85 & 92.63 & 93.13 & $1.66 \pm 0.13$ & $6.14 \pm 0.13$ \\
\hline & $4 \% / 4 \mathrm{~mm}$ & 89.98 & 94.61 & 94.16 & $0.53 \pm 0.24$ & $4.08 \pm 0.24$ \\
\hline & $3 \% / 1 \mathrm{~mm}$ & 64.30 & 83.54 & 77.17 & $6.37 \pm 0.23$ & $12.96 \pm 0.23$ \\
\hline \multirow[t]{4}{*}{ Brain 1} & $2 \% / 2 \mathrm{~mm}$ & 75.66 & 77.22 & 78.12 & $0.90 \pm 0.04$ & $0.64 \pm 0.04$ \\
\hline & $3 \% / 3 \mathrm{~mm}$ & 88.34 & 90.00 & 90.82 & $0.83 \pm 0.03$ & $0.83 \pm 0.03$ \\
\hline & $4 \% / 4 \mathrm{~mm}$ & 94.07 & 95.05 & 96.07 & $1.02 \pm 0.04$ & $-0.04 \pm 0.04$ \\
\hline & $3 \% / 1 \mathrm{~mm}$ & 67.85 & 70.01 & 69.79 & $0.21 \pm 0.01$ & $1.93 \pm 0.01$ \\
\hline \multirow[t]{4}{*}{ Liver } & $2 \% / 2 \mathrm{~mm}$ & 88.03 & 94.11 & 96.22 & $2.94 \pm 0.37$ & $3.11 \pm 0.37$ \\
\hline & $3 \% / 3 \mathrm{~mm}$ & 95.50 & 98.83 & 98.72 & $0.11 \pm 0.01$ & $3.19 \pm 0.01$ \\
\hline & $4 \% / 4 \mathrm{~mm}$ & 98.48 & 99.71 & 99.90 & $0.19 \pm 0.00$ & $1.02 \pm 0.00$ \\
\hline & $3 \% / 1 \mathrm{~mm}$ & 87.09 & 93.27 & 98.44 & $5.17 \pm 0.00$ & $0.97 \pm 0.00$ \\
\hline \multirow[t]{4}{*}{$H E B N 1$} & $2 \% / 2 \mathrm{~mm}$ & 70.43 & 71.52 & 71.79 & $0.28 \pm 0.13$ & $0.80 \pm 0.13$ \\
\hline & $3 \% / 3 \mathrm{~mm}$ & 82.60 & 83.33 & 83.81 & $0.49 \pm 0.19$ & $0.23 \pm 0.19$ \\
\hline & $4 \% / 4 \mathrm{~mm}$ & 90.12 & 90.72 & 90.80 & $0.15 \pm 0.12$ & $0.45 \pm 0.12$ \\
\hline & $3 \% / 1 \mathrm{~mm}$ & 57.23 & 58.78 & 59.90 & $1.13 \pm 0.02$ & $0.41 \pm 0.02$ \\
\hline \multirow{4}{*}{$\begin{array}{l}\text { Lung 2 - POST } \\
(P S Q A)\end{array}$} & $2 \% / 2 \mathrm{~mm}$ & 89.42 & 96.83 & 96.30 & $0.57 \pm 0.00$ & $6.84 \pm 0.00$ \\
\hline & $3 \% / 3 \mathrm{~mm}$ & 97.60 & 99.78 & 99.89 & $0.11 \pm 0.02$ & $2.02 \pm 0.02$ \\
\hline & $4 \% / 4 \mathrm{~mm}$ & 99.58 & 99.97 & 100.00 & $0.03 \pm 0.00$ & $0.34 \pm 0.00$ \\
\hline & $3 \% / 1 \mathrm{~mm}$ & 88.78 & 92.93 & 94.59 & $1.65 \pm 0.00$ & $2.45 \pm 0.00$ \\
\hline \multirow{4}{*}{$\begin{array}{l}\text { Lung } 2-L P O \\
(P S Q A)\end{array}$} & $2 \% / 2 \mathrm{~mm}$ & 92.03 & 97.75 & 99.02 & $1.27 \pm 0.00$ & $4.43 \pm 0.00$ \\
\hline & $3 \% / 3 \mathrm{~mm}$ & 98.14 & 99.51 & 100.01 & $0.50 \pm 0.00$ & $0.78 \pm 0.00$ \\
\hline & $4 \% / 4 \mathrm{~mm}$ & 99.66 & 99.97 & 100.01 & $0.03 \pm 0.00$ & $0.26 \pm 0.00$ \\
\hline & $3 \% / 1 \mathrm{~mm}$ & 92.72 & 96.26 & 99.19 & $2.92 \pm 0.00$ & $0.53 \pm 0.00$ \\
\hline \multirow[t]{4}{*}{ Lung 3} & $2 \% / 2 \mathrm{~mm}$ & 81.93 & 90.28 & 93.18 & $2.90 \pm 0.46$ & $5.40 \pm 0.46$ \\
\hline & $3 \% / 3 \mathrm{~mm}$ & 92.44 & 96.45 & 96.74 & $0.28 \pm 0.00$ & $3.67 \pm 0.00$ \\
\hline & $4 \% / 4 \mathrm{~mm}$ & 96.76 & 98.48 & 99.50 & $1.03 \pm 0.01$ & $0.63 \pm 0.01$ \\
\hline & $3 \% / 1 \mathrm{~mm}$ & 75.15 & 83.96 & 85.82 & $2.06 \pm 2.17$ & $6.69 \pm 2.17$ \\
\hline \multirow[t]{4}{*}{ Brain 2} & $2 \% / 2 \mathrm{~mm}$ & 90.68 & 92.71 & 95.27 & $2.56 \pm 0.04$ & $-0.55 \pm 0.04$ \\
\hline & $3 \% / 3 \mathrm{~mm}$ & 96.35 & 97.45 & 99.57 & $2.12 \pm 0.00$ & $-1.04 \pm 0.00$ \\
\hline & $4 \% / 4 \mathrm{~mm}$ & 98.53 & 99.06 & 99.67 & $0.61 \pm 0.04$ & $-0.10 \pm 0.04$ \\
\hline & $3 \% / 1 \mathrm{~mm}$ & 81.61 & 84.62 & 85.65 & $1.20 \pm 1.74$ & $1.79 \pm 1.74$ \\
\hline \multirow[t]{4}{*}{$H E N 2$} & $2 \% / 2 \mathrm{~mm}$ & 92.24 & 93.81 & 94.09 & $0.42 \pm 0.11$ & $1.15 \pm 0.11$ \\
\hline & $3 \% / 3 \mathrm{~mm}$ & 97.61 & 98.25 & 98.70 & $0.49 \pm 0.03$ & $0.14 \pm 0.03$ \\
\hline & $4 \% / 4 \mathrm{~mm}$ & 99.10 & 99.28 & 99.47 & $0.22 \pm 0.08$ & $-0.05 \pm 0.08$ \\
\hline & $3 \% / 1 \mathrm{~mm}$ & 83.74 & 85.88 & 85.66 & $0.22 \pm 0.02$ & $1.91 \pm 0.02$ \\
\hline
\end{tabular}

Table 3: Summary of the results for the $\mathrm{MC}_{\text {ref }}$ case when $\bar{\sigma}_{\min }=2 \%$. The true passing rates for $\bar{\sigma}=\bar{\sigma}_{\min }$ and $\bar{\sigma}=0.3 \%$ are shown, along with the predicted passing rate for $\bar{\sigma}=0.3 \%$. The two last columns show the absolute error made by predicting the GPR at $\bar{\sigma}=0.3 \%$ as well as the gain as defined in Eq. (8) (average \pm standard deviation). 


\begin{tabular}{|c|c|c|c|c|c|c|}
\hline Case & & $\begin{array}{c}\mathbf{G P R}_{\text {true, } \mathbf{1 \%}} \\
(\%) \\
\end{array}$ & $\begin{array}{c}\mathbf{G P R}_{\text {true }, 0.3 \%} \\
(\%)\end{array}$ & $\begin{array}{c}\mathbf{G P R}_{\text {predicted,0.3\% }} \\
(\%)\end{array}$ & $\begin{array}{c}\text { Absolute error } \\
(\%)\end{array}$ & $\begin{array}{c}\text { Gain } \\
(\%) \\
\end{array}$ \\
\hline \multirow[t]{4}{*}{ Prostate } & $2 \% / 2 \mathrm{~mm}$ & 95.99 & 91.00 & 92.88 & $1.89 \pm 0.77$ & $3.11 \pm 0.77$ \\
\hline & $3 \% / 3 \mathrm{~mm}$ & 99.43 & 98.31 & 99.36 & $1.05 \pm 0.10$ & $0.08 \pm 0.10$ \\
\hline & $4 \% / 4 \mathrm{~mm}$ & 99.68 & 99.60 & 99.68 & $0.08 \pm 0.00$ & $0.00 \pm 0.00$ \\
\hline & $3 \% / 1 \mathrm{~mm}$ & 88.45 & 86.97 & 86.87 & $0.45 \pm 0.60$ & $1.05 \pm 0.60$ \\
\hline \multirow[t]{4}{*}{ Lung 1} & $2 \% / 2 \mathrm{~mm}$ & 91.15 & 89.70 & 90.06 & $0.42 \pm 0.46$ & $1.03 \pm 0.46$ \\
\hline & $3 \% / 3 \mathrm{~mm}$ & 94.23 & 93.22 & 93.17 & $0.37 \pm 0.42$ & $0.65 \pm 0.42$ \\
\hline & $4 \% / 4 \mathrm{~mm}$ & 96.12 & 95.30 & 95.26 & $0.32 \pm 0.29$ & $0.50 \pm 0.29$ \\
\hline & $3 \% / 1 \mathrm{~mm}$ & 84.39 & 83.99 & 84.16 & $0.28 \pm 0.11$ & $0.10 \pm 0.11$ \\
\hline \multirow[t]{4}{*}{ Brain } & $2 \% / 2 \mathrm{~mm}$ & 79.65 & 74.97 & 74.07 & $1.50 \pm 1.22$ & $3.18 \pm 1.22$ \\
\hline & $3 \% / 3 \mathrm{~mm}$ & 90.24 & 86.88 & 87.43 & $0.76 \pm 0.59$ & $2.61 \pm 0.59$ \\
\hline & $4 \% / 4 \mathrm{~mm}$ & 93.74 & 91.68 & 92.46 & $0.87 \pm 0.58$ & $1.20 \pm 0.58$ \\
\hline & $3 \% / 1 \mathrm{~mm}$ & 69.63 & 68.15 & 67.72 & $0.82 \pm 0.58$ & $0.66 \pm 0.58$ \\
\hline \multirow[t]{4}{*}{ Liver } & $2 \% / 2 \mathrm{~mm}$ & 96.84 & 95.40 & 96.75 & $1.35 \pm 0.08$ & $0.10 \pm 0.08$ \\
\hline & $3 \% / 3 \mathrm{~mm}$ & 99.00 & 98.76 & 98.99 & $0.23 \pm 0.00$ & $0.00 \pm 0.00$ \\
\hline & $4 \% / 4 \mathrm{~mm}$ & 99.37 & 99.33 & 99.37 & $0.04 \pm 0.00$ & $0.00 \pm 0.00$ \\
\hline & $3 \% / 1 \mathrm{~mm}$ & 93.14 & 93.45 & 93.68 & $0.27 \pm 0.01$ & $0.04 \pm 0.01$ \\
\hline \multirow[t]{4}{*}{$H E N$} & $2 \% / 2 \mathrm{~mm}$ & 67.98 & 65.64 & 64.59 & $1.00 \pm 0.30$ & $1.39 \pm 0.30$ \\
\hline & $3 \% / 3 \mathrm{~mm}$ & 79.72 & 76.83 & 76.54 & $0.65 \pm 0.91$ & $2.31 \pm 0.91$ \\
\hline & $4 \% / 4 \mathrm{~mm}$ & 87.20 & 84.23 & 84.53 & $0.45 \pm 1.08$ & $2.59 \pm 1.08$ \\
\hline & $3 \% / 1 \mathrm{~mm}$ & 56.24 & 55.22 & 54.49 & $0.75 \pm 0.28$ & $0.30 \pm 0.28$ \\
\hline \multirow{4}{*}{$\begin{array}{l}\text { Lung } 2 \text { - POST } \\
\text { (PSQA) }\end{array}$} & $2 \% / 2 \mathrm{~mm}$ & 99.98 & 99.79 & 99.97 & $0.18 \pm 0.00$ & $0.00 \pm 0.00$ \\
\hline & $3 \% / 3 \mathrm{~mm}$ & 100.00 & 100.00 & 100.00 & $0.00 \pm 0.00$ & $-0.00 \pm 0.00$ \\
\hline & $4 \% / 4 \mathrm{~mm}$ & 100.00 & 100.00 & 100.00 & $0.00 \pm 0.00$ & $-0.00 \pm 0.00$ \\
\hline & $3 \% / 1 \mathrm{~mm}$ & 98.15 & 98.18 & 98.33 & $0.15 \pm 0.01$ & $-0.11 \pm 0.01$ \\
\hline \multirow{4}{*}{$\begin{array}{l}\text { Lung } 2-L P O \\
(P S Q A)\end{array}$} & $2 \% / 2 \mathrm{~mm}$ & 100.00 & 99.97 & 100.00 & $0.03 \pm 0.00$ & $0.00 \pm 0.00$ \\
\hline & $3 \% / 3 \mathrm{~mm}$ & 100.00 & 100.00 & 100.00 & $0.00 \pm 0.00$ & $-0.00 \pm 0.00$ \\
\hline & $4 \% / 4 \mathrm{~mm}$ & 100.00 & 100.00 & 100.00 & $0.00 \pm 0.00$ & $-0.00 \pm 0.00$ \\
\hline & $3 \% / 1 \mathrm{~mm}$ & 99.55 & 99.74 & 99.73 & $0.01 \pm 0.00$ & $0.18 \pm 0.00$ \\
\hline \multirow[t]{4}{*}{ Lung 3} & $2 \% / 2 \mathrm{~mm}$ & 97.11 & 96.53 & 97.05 & $0.52 \pm 0.04$ & $0.06 \pm 0.04$ \\
\hline & $3 \% / 3 \mathrm{~mm}$ & 99.31 & 99.23 & 99.31 & $0.07 \pm 0.00$ & $0.00 \pm 0.00$ \\
\hline & $4 \% / 4 \mathrm{~mm}$ & 99.81 & 99.77 & 99.80 & $0.03 \pm 0.00$ & $0.00 \pm 0.00$ \\
\hline & $3 \% / 1 \mathrm{~mm}$ & 88.75 & 88.49 & 88.74 & $0.25 \pm 0.00$ & $0.00 \pm 0.00$ \\
\hline \multirow[t]{4}{*}{ Brain 2} & $2 \% / 2 \mathrm{~mm}$ & 98.64 & 97.59 & 98.32 & $0.74 \pm 0.14$ & $0.32 \pm 0.14$ \\
\hline & $3 \% / 3 \mathrm{~mm}$ & 99.97 & 99.92 & 99.96 & $0.04 \pm 0.00$ & $0.00 \pm 0.00$ \\
\hline & $4 \% / 4 \mathrm{~mm}$ & 100.00 & 100.00 & 100.00 & $0.00 \pm 0.00$ & $-0.00 \pm 0.00$ \\
\hline & $3 \% / 1 \mathrm{~mm}$ & 90.32 & 88.89 & 89.25 & $0.36 \pm 0.32$ & $1.07 \pm 0.32$ \\
\hline \multirow[t]{4}{*}{$H E S N 2$} & $2 \% / 2 \mathrm{~mm}$ & 95.13 & 95.02 & 95.09 & $0.07 \pm 0.00$ & $0.03 \pm 0.00$ \\
\hline & $3 \% / 3 \mathrm{~mm}$ & 98.98 & 98.93 & 98.97 & $0.04 \pm 0.00$ & $0.01 \pm 0.00$ \\
\hline & $4 \% / 4 \mathrm{~mm}$ & 99.74 & 99.71 & 99.73 & $0.02 \pm 0.00$ & $0.00 \pm 0.00$ \\
\hline & $3 \% / 1 \mathrm{~mm}$ & 86.15 & 86.21 & 86.56 & $0.35 \pm 0.00$ & $-0.30 \pm 0.00$ \\
\hline
\end{tabular}

Table 4: Summary of the results for the $\mathrm{MC}_{\text {eval }}$ case when $\bar{\sigma}_{\min }=1 \%$. The true passing rates for $\bar{\sigma}=\bar{\sigma}_{\min }$ and $\bar{\sigma}=0.3 \%$ are shown, along with the predicted passing rate for $\bar{\sigma}=0.3 \%$. The two last columns show the absolute error made by predicting the GPR at $\bar{\sigma}=0.3 \%$ as well as the gain as defined in Eq. (8) (average \pm standard deviation). 


\begin{tabular}{|c|c|c|c|c|c|c|}
\hline Case & & $\begin{array}{c}\mathbf{G P R}_{\text {true, } \mathbf{2 \%}} \\
(\%) \\
\end{array}$ & $\begin{array}{c}\mathbf{G P R}_{\text {true, } \mathbf{0 . 3 \%}} \\
(\%)\end{array}$ & $\begin{array}{c}\mathbf{G P R}_{\text {predicted,0.3\% }} \\
(\%)\end{array}$ & $\begin{array}{c}\text { Absolute error } \\
(\%)\end{array}$ & $\begin{array}{c}\text { Gain } \\
(\%) \\
\end{array}$ \\
\hline \multirow[t]{4}{*}{ Prostate } & $2 \% / 2 \mathrm{~mm}$ & 98.62 & 91.00 & 97.23 & $6.24 \pm 1.58$ & $1.46 \pm 1.58$ \\
\hline & $3 \% / 3 \mathrm{~mm}$ & 99.62 & 98.31 & 99.60 & $1.30 \pm 0.01$ & $0.02 \pm 0.01$ \\
\hline & $4 \% / 4 \mathrm{~mm}$ & 99.71 & 99.60 & 99.70 & $0.11 \pm 0.00$ & $0.00 \pm 0.00$ \\
\hline & $3 \% / 1 \mathrm{~mm}$ & 90.66 & 86.97 & 87.90 & $1.34 \pm 1.12$ & $2.39 \pm 1.12$ \\
\hline \multirow[t]{4}{*}{ Lung 1} & $2 \% / 2 \mathrm{~mm}$ & 92.78 & 89.70 & 90.50 & $1.06 \pm 0.73$ & $2.04 \pm 0.73$ \\
\hline & $3 \% / 3 \mathrm{~mm}$ & 95.62 & 93.22 & 93.63 & $1.31 \pm 0.78$ & $1.12 \pm 0.78$ \\
\hline & $4 \% / 4 \mathrm{~mm}$ & 97.06 & 95.30 & 96.15 & $1.26 \pm 0.41$ & $0.52 \pm 0.41$ \\
\hline & $3 \% / 1 \mathrm{~mm}$ & 84.68 & 83.99 & 84.90 & $0.90 \pm 0.00$ & $-0.21 \pm 0.00$ \\
\hline \multirow[t]{4}{*}{ Brain } & $2 \% / 2 \mathrm{~mm}$ & 86.35 & 74.97 & 76.99 & $2.06 \pm 3.25$ & $9.36 \pm 3.25$ \\
\hline & $3 \% / 3 \mathrm{~mm}$ & 93.15 & 86.88 & 90.19 & $3.37 \pm 0.84$ & $2.91 \pm 0.84$ \\
\hline & $4 \% / 4 \mathrm{~mm}$ & 95.36 & 91.68 & 93.68 & $2.00 \pm 0.49$ & $1.69 \pm 0.49$ \\
\hline & $3 \% / 1 \mathrm{~mm}$ & 72.66 & 68.15 & 67.72 & $0.84 \pm 1.09$ & $3.68 \pm 1.09$ \\
\hline \multirow[t]{4}{*}{ Liver } & $2 \% / 2 \mathrm{~mm}$ & 97.36 & 95.40 & 97.15 & $1.75 \pm 0.16$ & $0.21 \pm 0.16$ \\
\hline & $3 \% / 3 \mathrm{~mm}$ & 99.06 & 98.76 & 99.05 & $0.29 \pm 0.01$ & $0.01 \pm 0.01$ \\
\hline & $4 \% / 4 \mathrm{~mm}$ & 99.43 & 99.33 & 99.40 & $0.07 \pm 0.02$ & $0.02 \pm 0.02$ \\
\hline & $3 \% / 1 \mathrm{~mm}$ & 93.14 & 93.45 & 93.88 & $0.43 \pm 0.00$ & $0.49 \pm 0.00$ \\
\hline \multirow[t]{4}{*}{$H E N$} & $2 \% / 2 \mathrm{~mm}$ & 72.74 & 65.64 & 65.16 & $0.97 \pm 1.44$ & $6.20 \pm 1.44$ \\
\hline & $3 \% / 3 \mathrm{~mm}$ & 84.49 & 76.83 & 76.84 & $0.76 \pm 1.23$ & $6.98 \pm 1.23$ \\
\hline & $4 \% / 4 \mathrm{~mm}$ & 91.08 & 84.23 & 85.52 & $1.75 \pm 1.66$ & $5.17 \pm 1.66$ \\
\hline & $3 \% / 1 \mathrm{~mm}$ & 58.79 & 55.22 & 53.81 & $1.48 \pm 0.90$ & $2.13 \pm 0.90$ \\
\hline \multirow{4}{*}{$\begin{array}{l}\text { Lung } 2 \text { - POST } \\
\text { (PSQA) }\end{array}$} & $2 \% / 2 \mathrm{~mm}$ & 99.99 & 99.79 & 99.86 & $0.08 \pm 0.08$ & $0.11 \pm 0.08$ \\
\hline & $3 \% / 3 \mathrm{~mm}$ & 100.00 & 100.00 & 100.00 & $0.00 \pm 0.00$ & $-0.00 \pm 0.00$ \\
\hline & $4 \% / 4 \mathrm{~mm}$ & 100.00 & 100.00 & 100.00 & $0.00 \pm 0.00$ & $-0.00 \pm 0.00$ \\
\hline & $3 \% / 1 \mathrm{~mm}$ & 97.93 & 98.18 & 98.65 & $0.47 \pm 0.29$ & $-0.23 \pm 0.29$ \\
\hline \multirow{4}{*}{$\begin{array}{l}\text { Lung } 2-L P O \\
(P S Q A)\end{array}$} & $2 \% / 2 \mathrm{~mm}$ & 100.00 & 99.97 & 100.00 & $0.03 \pm 0.00$ & $-0.00 \pm 0.00$ \\
\hline & $3 \% / 3 \mathrm{~mm}$ & 100.00 & 100.00 & 100.00 & $0.00 \pm 0.00$ & $-0.00 \pm 0.00$ \\
\hline & $4 \% / 4 \mathrm{~mm}$ & 100.00 & 100.00 & 100.00 & $0.00 \pm 0.00$ & $-0.00 \pm 0.00$ \\
\hline & $3 \% / 1 \mathrm{~mm}$ & 99.34 & 99.74 & 99.37 & $0.36 \pm 0.01$ & $0.04 \pm 0.01$ \\
\hline \multirow[t]{4}{*}{ Lung 3} & $2 \% / 2 \mathrm{~mm}$ & 97.44 & 96.53 & 97.28 & $0.74 \pm 0.10$ & $0.16 \pm 0.10$ \\
\hline & $3 \% / 3 \mathrm{~mm}$ & 99.39 & 99.23 & 99.36 & $0.13 \pm 0.03$ & $0.03 \pm 0.03$ \\
\hline & $4 \% / 4 \mathrm{~mm}$ & 99.85 & 99.77 & 99.83 & $0.05 \pm 0.02$ & $0.02 \pm 0.02$ \\
\hline & $3 \% / 1 \mathrm{~mm}$ & 88.85 & 88.49 & 88.33 & $0.19 \pm 0.53$ & $0.16 \pm 0.53$ \\
\hline \multirow[t]{4}{*}{ Brain 2} & $2 \% / 2 \mathrm{~mm}$ & 99.12 & 97.59 & 98.74 & $1.18 \pm 0.12$ & $0.35 \pm 0.12$ \\
\hline & $3 \% / 3 \mathrm{~mm}$ & 99.98 & 99.92 & 99.98 & $0.06 \pm 0.00$ & $-0.00 \pm 0.00$ \\
\hline & $4 \% / 4 \mathrm{~mm}$ & 100.00 & 100.00 & 100.00 & $0.00 \pm 0.00$ & $-0.00 \pm 0.00$ \\
\hline & $3 \% / 1 \mathrm{~mm}$ & 91.74 & 88.89 & 88.80 & $0.61 \pm 0.66$ & $2.24 \pm 0.66$ \\
\hline \multirow[t]{4}{*}{$H \& N^{2}$} & $2 \% / 2 \mathrm{~mm}$ & 95.37 & 95.02 & 95.26 & $0.24 \pm 0.04$ & $0.11 \pm 0.04$ \\
\hline & $3 \% / 3 \mathrm{~mm}$ & 99.08 & 98.93 & 98.77 & $0.16 \pm 0.18$ & $-0.01 \pm 0.18$ \\
\hline & $4 \% / 4 \mathrm{~mm}$ & 99.79 & 99.71 & 99.51 & $0.20 \pm 0.22$ & $-0.13 \pm 0.22$ \\
\hline & $3 \% / 1 \mathrm{~mm}$ & 85.57 & 86.21 & 86.20 & $0.11 \pm 0.09$ & $0.53 \pm 0.09$ \\
\hline
\end{tabular}

Table 5: Summary of the results for the $\mathrm{MC}_{\text {eval }}$ case when $\bar{\sigma}_{\min }=2 \%$. The true passing rates for $\bar{\sigma}=\bar{\sigma}_{\min }$ and $\bar{\sigma}=0.3 \%$ are shown, along with the predicted passing rate for $\bar{\sigma}=0.3 \%$. The two last columns show the absolute error made by predicting the GPR at $\bar{\sigma}=0.3 \%$ as well as the gain as defined in Eq. (8) (average \pm standard deviation). 